\title{
Estimating the location of the open-closed magnetic field line boundary from auroral images
}

\author{
N. Longden ${ }^{1}$, G. Chisham ${ }^{1}$, M. P. Freeman ${ }^{1}$, G. A. Abel ${ }^{1}$, and T. Sotirelis ${ }^{2}$ \\ ${ }^{1}$ British Antarctic Survey, High Cross, Madingley Road, Cambridge, CB3 0ET, UK \\ ${ }^{2}$ The Johns Hopkins University, Applied Physics Laboratory, Laurel, MD 20723, USA \\ Received: 12 March 2010 - Revised: 7 July 2010 - Accepted: 27 August 2010 - Published: 10 September 2010
}

\begin{abstract}
The open-closed magnetic field line boundary (OCB) delimits the region of open magnetic flux forming the polar cap in the Earth's ionosphere. We present a reliable, automated method for determining the location of the poleward auroral luminosity boundary (PALB) from far ultraviolet (FUV) images of the aurora, which we use as a proxy for the OCB. This technique models latitudinal profiles of auroral luminosity as both a single and double Gaussian function with a quadratic background to produce estimates of the PALB without prior knowledge of the level of auroral activity or of the presence of bifurcation in the auroral oval. We have applied this technique to FUV images recorded by the IMAGE satellite from May 2000 until August 2002 to produce a database of over a million PALB location estimates, which is freely available to download. From this database, we assess and illustrate the accuracy and reliability of this technique during varying geomagnetic conditions. We find that up to $35 \%$ of our PALB estimates are made from double Gaussian fits to latitudinal intensity profiles, in preference to single Gaussian fits, in nightside magnetic local time (MLT) sectors. The accuracy of our PALBs as a proxy for the location of the OCB is evaluated by comparison with particle precipitation boundary (PPB) proxies from the DMSP satellites. We demonstrate the value of this technique in estimating the total rate of magnetic reconnection from the time variation of the polar cap area calculated from our OCB estimates.
\end{abstract}

Keywords. Magnetospheric physics (Auroral phenomena; Magnetospheric configuration and dynamics; Instruments and techniques)

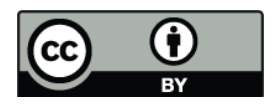

Correspondence to: $\mathrm{N}$. Longden (nilo@bas.ac.uk)

\section{Introduction}

The polar cap is the ionospheric footprint of the region of open magnetic flux in the Earth's magnetosphere, i.e., those magnetic field lines that attach both to the Earth and to the interplanetary magnetic field (IMF). The interface between the open field lines of the polar cap and the closed field lines of the auroral zone of the ionosphere is termed the openclosed boundary (OCB). Changes in the area of the polar cap are linked to magnetic reconnection, with the addition of open flux as a result of reconnection between the geomagnetic field lines and IMF on the dayside and the closure of flux on the nightside in the magnetotail (e.g., Siscoe and Huang, 1985; Brittnacher et al., 1999). Hence, the rate of change of polar cap area (PCA) can be used as a proxy for the total net rate of magnetic reconnection (e.g., Milan et al., 2003; Chisham et al., 2008). Also, the local rate of reconnection and its spatial variability can be determined wherever the OCB location is known and measurements of ionospheric plasma flow across it exist, allowing a local estimation of the rate of flux transfer between open and closed field line regions (e.g., Pinnock et al., 2003; Chisham et al., 2004; Hubert et al., 2006; Chisham et al., 2008). Therefore, accurate determination of the OCB location is a prerequisite to estimation of the rate of reconnection.

The location of the OCB can be best estimated from measurements of particle precipitation boundaries (PPB) made by satellites in low-altitude orbits (e.g., Vampola, 1971; Makita et al., 1983; Makita and Meng, 1984; Newell et al., 1991; Mishin et al., 1992; Newell et al., 1996; Sotirelis and Newell, 2000). However, whilst these PPBs are considered to provide the best proxy of the OCB, they typically provide only infrequent, point measurements of this boundary. Many other instruments have been used to provide estimates of the location of the OCB with higher

Published by Copernicus Publications on behalf of the European Geosciences Union. 
spatial and temporal coverage. These include HF radars (e.g., Baker et al., 1995, 1997; Milan et al., 1999; Milan and Lester, 2001; Chisham et al., 2001, 2002; Chisham and Freeman, 2003), ground-based magnetometers (e.g., Iijima and Potemra, 1978; Mishin, 1990), all-sky cameras (e.g., Akasofu and Kimball, 1965; Feldstein and Galperin, 1985), meridian scanning photometers (e.g., Blanchard et al., 1995; Sandholt et al., 1998), and satellite-based imagers (e.g., Brittnacher et al., 1999; Kauristie et al., 1999; Baker et al., 2000; Carbary et al., 2003; Boakes et al., 2008). Arguably the best instrument to estimate the location of the complete OCB (in a single hemisphere) is the satellite-based imager, which can image the whole auroral oval at a time resolution of the order of minutes, for hours at a time (see e.g., Boakes et al., 2008).

Since the earliest auroral imager observations, a number of different techniques have been used to estimate the poleward boundary of auroral luminosity, thought to provide a good proxy for the location of the OCB. These techniques have included visual inspection (e.g., Elphinstone et al., 1990; Gjerloev et al., 2007), identifying a return to a threshold of low auroral luminosity poleward of the main emission (e.g., Brittnacher et al., 1999; Baker et al., 2000), identifying a return to a fraction of the peak intensity on the poleward edge of the main emission (e.g., Kauristie et al., 1999; Baker et al., 2000; Carbary et al., 2003; Boakes et al., 2008), and identifying a return to an intensity threshold relative to background intensity levels (e.g., Mende et al., 2003; Gjerloev et al., 2008). Carbary et al. (2003) estimated over 23000 poleward auroral luminosity boundaries (PALB) from images recorded by the ultraviolet imager (UVI) on the Polar satellite, by modelling latitudinal profiles of auroral luminosity intensity as a Gaussian function superimposed on a quadratic background. This method assumes that the auroral oval is characterised by a single, contiguous band of emission across magnetic latitudes, for all magnetic local time (MLT) sectors, at all times.

During substorm recovery, the auroral oval often exhibits a "double oval" configuration with a main oval and a poleward arc of emission (Elphinstone et al., 1995a,b). Gjerloev et al. (2008) have confirmed the configuration of a bifurcated auroral oval in UV images during substorm activity through a superposed epoch analysis of 116 isolated substorms. A function with two Gaussian components has been used to model latitudinal profiles of auroral intensity during substorm activity when bifurcation may be present in the oval (Gjerloev et al., 2008; Mende et al., 2003). Gjerloev et al. (2008) consider the Gaussian peaks representing the main oval and the bulge of the poleward emission to be quasi-independent phenomena, arising from bifurcation in the particle precipitation or energisation regions in the magnetosphere. When a double oval configuration exists, the poleward arc maps close to the OCB, at least in the nightside ionosphere (Elphinstone et al., 1995a). Accurate modelling of the auroral luminosity intensity profile during a double oval configuration is required for the best estimation of the OCB location, particularly during substorm recovery when this configuration is prevalent (Elphinstone et al., 1995b), although substorms occurring during ongoing geomagnetic activity do not always exhibit the classical evolution of isolated substorms with auroral bifurcation (Hoffman et al., 2010).

The accuracy of the PALB as a proxy for the OCB has been evaluated by comparison with satellite determined PPBs (e.g., Kauristie et al., 1999; Baker et al., 2000; Carbary et al., 2003; Boakes et al., 2008). These studies have highlighted systematic differences between the locations of the PPBs and PALBs and that this discrepancy varies with MLT. The comparative studies of Kauristie et al. (1999), Baker et al. (2000), and Carbary et al. (2003) estimated PALBs from a single ultraviolet imager based on either the Viking or Polar satellite. Boakes et al. (2008) estimated PALBs from FUV images recorded by the IMAGE satellite. The IMAGE FUV instrument has three detectors enabling the discrimination between auroral emissions resulting from proton and electron precipitation. Boakes et al. (2008) showed that the PALBs derived from the FUV detector that is sensitive to proton emissions were more poleward than those derived from the detectors that are sensitive to electron emissions in pre-dawn MLT sectors, and were located equatorward during the dusk to premidnight MLT sectors. Boakes et al. (2008) were able to improve the correlation between PPBs and PALBs in some MLT sectors by selecting boundaries from the most appropriate FUV detector for that sector, for example, selecting boundaries from the detector sensitive to proton emission from 01:00 to 07:00 MLT.

In this paper, we present an automated method to estimate poleward and equatorward auroral luminosity boundaries (EALB) from IMAGE FUV images and show how to correct the PALBs for use as a proxy for the OCB. Our technique brings together the strengths of various other methods. We present analysis of a database of over 1 million PALB location estimates derived from IMAGE FUV images using this technique, and demonstrate how satellite-measured PPBs can be used to improve the accuracy of these boundaries as a proxy for the OCB. The OCB location estimates can be used to compile an extensive set of reconnection rate estimations, enabling further analysis of this fundamental physical process.

\section{Instrumentation}

\subsection{IMAGE FUV}

The IMAGE FUV instrument is comprised of three detectors; the Wideband Imaging Camera (WIC) and two Spectrographic Imagers (SI12 and SI13) (Mende et al., 2000b). The WIC sensor has a passband of 140 to $190 \mathrm{~nm}$, observing emissions from the $\mathrm{N}_{2}$ Lyman-Birge-Hopfield band (Mende et al., 2000a,b). The SI12 detector is sensitive to Dopplershifted Lyman- $\alpha$ emissions in the $121.8 \mathrm{~nm}$ band, caused by proton precipitation (Mende et al., 2000b,c). The SI13 detector is sensitive to the $135.6 \mathrm{~nm}$ oxygen emission band 
resulting from energetic electron precipitation (Mende et al., $2000 \mathrm{~b}, \mathrm{c})$. FUV images are recorded with a time resolution of two minutes, determined by the spin period of the satellite (e.g., Burch, 2000). Both SI detectors produce images with a $128 \times 128$ pixel resolution while the WIC detector has a resolution of $256 \times 256$ pixels (Mende et al., 2000b). During its operation (years 2000 to 2005), the IMAGE satellite was in an elliptical orbit at $90^{\circ}$ inclination with an altitude of 7 Earth radii $\left(R_{\mathrm{E}}\right)$ at apogee and $1000 \mathrm{~km}$ at perigee, and with an orbital period of $13.5 \mathrm{~h}$ (e.g., Burch, 2000; Gibson et al., 2000; Mende et al., 2000b). IMAGE FUV data are available from May 2000 until December 2005. We use the two years of data from the start of the mission until August 2002, while the satellite apogee is close to the north pole (e.g., Frey and Mende, 2006). Some inaccuracy exists in the pointing information of the IMAGE satellite, with systematic errors of up to 4 pixels in the spin axis plane and 2 pixels perpendicular to the spin plane (e.g., Frey et al., 2004; Frey and Mende, 2006). According to Frey et al. (2004), this pointing error can increase the positional uncertainty of local time determination during summer and winter periods and uncertainty in latitude determination during the spring and autumn.

\subsection{DMSP}

PPBs are taken from the SSJ/4 precipitating electron and ion spectrometer instruments onboard the low-altitude Defense Meteorological Satellites Program (DMSP) satellites. The DMSP satellites are located in a sun-synchronous polar orbit at an altitude of around $830 \mathrm{~km}$. Their orbital period is $101 \mathrm{~min}$. The SSJ/4 particle detectors cover an energy range of $30 \mathrm{eV}$ to $30 \mathrm{keV}$ across twenty channels (Hardy et al., 1984). During the period of May 2000 until December 2001, satellites DMSP 11 to 15 were in operation. The algorithm used for automated identification of nightside PPBs from the DMSP energy spectra is detailed in Newell et al. (1996) while the algorithm to determine dayside PPBs is outlined in Sotirelis and Newell (2000), based on Newell et al. (1991). We use the b6 boundary representing the poleward edge of subvisual drizzle as the proxy for the OCB in nightside MLT sectors and the doc boundary, representing a transition from closed to open precipitation regions, in dayside MLT sectors. We refer to the nightside b6 boundary and the dayside doc boundary, derived from DMSP data, collectively as poleward auroral precipitation boundaries (PAPB). Where multiple crossings of the same magnetospheric region are identified in a single pass of the satellite, the most poleward boundary for each region is selected, and where clear transitions between precipitation regions cannot be made, PPBs are discarded (Sotirelis and Newell, 2000). Consequently regions void of particle precipitation equatorward of closed regions are considered to also be closed. Alternative classification schemes could be used but authors differ on the physical interpretation of spatially-separated void regions (Brittnacher et al., 1999). Due to the satellite orbit, Northern Hemi- sphere auroral boundaries are only available in the 05:00 to 22:00 MLT sectors during the time period under consideration.

\section{Boundary location technique}

\subsection{Boundary derivation}

In order to estimate the location of the auroral luminosity boundaries, we first determine latitudinal profiles of FUV intensity for each image from WIC, SI12 and SI13. The geomagnetic coordinates of the FUV images are transformed to altitude-adjusted corrected geomagnetic coordinates (AACGM, Baker and Wing, 1989), with an effective emission altitude of $130 \mathrm{~km}$, for consistency with the magnetic coordinate system used for DMSP data. For each image, 24 latitudinal emission intensity profiles are constructed by averaging available emission intensities in bins of $1^{\circ}$ magnetic latitude and $1 \mathrm{~h}$ of MLT. The luminosity intensity profiles are created covering the $50^{\circ}$ to $90^{\circ}$ AACGM latitude range (where satellite coverage is available).

Two functions are fit to each latitudinal profile; a single Gaussian function with a quadratic component (Eq. 1) and a double Gaussian function with a quadratic component (Eq. 2),

$$
\begin{aligned}
F_{\mathrm{s}}(\lambda)=A_{0} \exp \left[-\frac{\left(\lambda-\mu_{0}\right)^{2}}{2 \sigma_{0}^{2}}\right]+B+C \lambda+D \lambda^{2} \\
F_{\mathrm{d}}(\lambda)=A_{\mathrm{e}} \exp \left[-\frac{\left(\lambda-\mu_{\mathrm{e}}\right)^{2}}{2 \sigma_{\mathrm{e}}^{2}}\right]+ \\
A_{\mathrm{p}} \exp \left[-\frac{\left(\lambda-\mu_{\mathrm{p}}\right)^{2}}{2 \sigma_{\mathrm{p}}^{2}}\right]+E+F \lambda+G \lambda^{2}
\end{aligned}
$$

where $\lambda$ is the magnetic latitude. In Eq. (1), $A_{0}, \mu_{0}$, and $\sigma_{0}$ are the peak amplitude, peak latitude, and width of the Gaussian, respectively, and $B, C$, and $D$, are the coefficients of the quadratic background of the function. In Eq. (2), $A_{\mathrm{e}}$, $\mu_{\mathrm{e}}$, and $\sigma_{\mathrm{e}}$ are the coefficients of the most equatorward of the two Gaussian components of the function, while $A_{\mathrm{p}}, \mu_{\mathrm{p}}$, and $\sigma_{\mathrm{p}}$ are the coefficients of the most poleward of the two Gaussian components. $E, F$, and $G$ are coefficients of the quadratic background of the function. In both cases, the Gaussian components of the function attempt to categorise auroral emissions while the quadratic components represent the background emissions. Carbary et al. (2003) and Boakes et al. (2008) also fit a function of the form of Eq. (1) to latitudinal intensity profiles in order to derive estimates for the location of the PALB while Mende et al. (2003) fit a function similar to Eq. (2) for this purpose.

WIC and SI13 images can be susceptible to contamination by dayglow in the FUV spectrum (e.g. Boakes et al., 2008; Hubert et al., 2006). Dayglow emission is generated by incident solar radiation on the atmosphere and is dependent 
on the solar zenith angle, with the greatest dayglow emission observable at the subsolar point (e.g., Meier, 1991, and references therein). When present, dayglow can be seen in latitudinal luminosity intensity profiles as very high intensity at the lowest magnetic latitudes, rapidly decreasing towards higher latitudes. Dayglow can be modelled by the quadratic background of the functions $F_{\mathrm{s}}(\lambda)$ and $F_{\mathrm{d}}(\lambda)$ (Carbary et al., 2003). However, during fitting, sometimes a Gaussian component of the functions will be fit to the dayglow in preference to the auroral emission (e.g., Boakes et al., 2008). In this case, the resulting PALB will be incorrectly located and should not be used as an estimate for the OCB.

We attempt to enforce the fitting of the Gaussian components of our models to the auroral emissions rather than the dayglow by constraining the lower limit of dayside luminosity intensity profiles (i.e., those in the 06:00 to 18:00 MLT sectors). Hence, we search the intensity profile to find the most equatorward local minimum in the $50^{\circ}$ to $90^{\circ}$ AACGM range to locate the latitude at which auroral emissions exceed the background. We then attempt to fit Eqs. (1) and (2) to the intensity profile from the latitude of this local minimum up to $90^{\circ}$. When a local minimum cannot be found, no attempt at fitting is made for that profile and no auroral luminosity boundary estimates are made. As the intensity profiles are typically noisy, we smooth the profiles using a boxcar sliding average and find the location of the local minima from the smoothed profiles. For intensity profiles derived from WIC images, a width of three points is used in the sliding average. A width of seven points is used for intensity profiles derived from SI13 images due to the lower resolution and count numbers of these images. The fits are subsequently made to the original, unsmoothed, intensity profiles. No dayglow removal is performed for intensity profiles covering nightside MLTs and those obtained from SI12 images at all local times, as these images are much less susceptible to dayglow contamination (e.g. Boakes et al., 2008; Hubert et al., 2006). Techniques do exist to pre-process FUV images in an attempt to completely remove dayglow by using an empirical reference model of dayglow emission from FUV images obtained during quiet conditions that can then be used to characterise dayglow in other images (Immel et al., 2000). While this technique has been applied to IMAGE FUV images (e.g., Meurant et al., 2003; DeJong et al., 2007), there is an impact on the extent of automation that can be achieved in the selection of appropriate quiet time images and a dependence between images is introduced. Some uncertainty is also introduced through the accuracy of the reference model in representing the actual dayglow in each specific image. Hence, for our purposes our basic dayglow mitigation method provides the best solution.

The Levenberg-Marquardt method for nonlinear leastsquares fitting, adapted from Press et al. (1992), is used to fit the functions $F_{\mathrm{s}}(\lambda)$ and $F_{\mathrm{d}}(\lambda)$ to each latitudinal luminosity intensity profile. Prior to fitting, initial values for the function parameters are estimated from the intensity pro- file. Specifically, the quadratic coefficients $D$ and $G$ are initially set to zero and initial estimates for the constant (i.e., $B$ and $E$ ) and linear (i.e., $C$ and $F$ ) coefficients are obtained by performing a linear least-squares fit to the intensity profile. The background intensity components with these initial values are subtracted from the intensity profile to produce an estimate for the auroral emissions. The location and magnitude of the one (or two) maxima in this derived auroral emission profile are selected as the initial estimates for the centre $(\mu)$ and amplitude $(A)$ of the Gaussian component(s) of $F_{\mathrm{S}}(\lambda)$ (or $F_{\mathrm{d}}(\lambda)$ ). Initial estimates for the widths $(\sigma)$ of the Gaussian components of $F_{\mathrm{S}}(\lambda)$ and $F_{\mathrm{d}}(\lambda)$ are made from estimates of the corresponding full width at half maximum (FWHM), where FWHM $=2 \sqrt{2 \ln (2)} \sigma$. The FWHM is estimated for each peak by calculating the distance between the points where the intensity has fallen to one half of the peak value on both sides of the peak. When a local minimum in a profile is found prior to the half maximum value being reached, the FWHM is estimated to this turning point.

Once estimates have been made for the function parameters, the Levenberg-Marquardt fit is iterated until either improvements in the fitting are negligible in successive iterations or a set maximum number of iterations is reached. We consider improvements in fitting between iterations to be negligible when the value of the reduced chi-squared goodness-of-fit statistic $\left(\chi^{2} / v\right.$, where $v$ is the degrees of freedom) reduces by less than 0.01 between successive iterations. We define the maximum number of iterations to be 200 as more than $97 \%$ of fits showing convergence have achieved full convergence to the optimum solution by this point. During fitting, we prevent the parameters relating to the Gaussian coefficients from becoming negative between successive iterations by inverting the sign of a negative increment that would move the parameter value below zero. This prevents the fitting of an inverted Gaussian profile to represent the separation of emission in a bifurcated intensity profile, for example.

The parameters returned by the final fitting iteration are used to estimate the EALB $\left(\lambda_{\mathrm{e}}\right)$ and PALB $\left(\lambda_{\mathrm{p}}\right)$. Following Carbary et al. (2003), we use the FWHM of the fitted Gaussian peaks offset from the centre of the peak, i.e.,

$\lambda_{\mathrm{e}}=\mu_{\mathrm{e}}-2 \sqrt{2 \ln (2)} \sigma_{\mathrm{e}}$
$\lambda_{\mathrm{p}}=\mu_{\mathrm{p}}+2 \sqrt{2 \ln (2)} \sigma_{\mathrm{p}}$

where $\mu_{\mathrm{e}}=\mu_{\mathrm{p}}=\mu_{0}$ and $\sigma_{\mathrm{e}}=\sigma_{\mathrm{p}}=\sigma_{0}$ for a single Gaussian profile. Uncertainties in the boundary locations can be derived according to Eq. (5),

$U_{\lambda}=\sqrt{\left(\frac{\partial \lambda}{\partial \mu} U_{\mu}\right)^{2}+\left(\frac{\partial \lambda}{\partial \sigma} U_{\sigma}\right)^{2}+2 \frac{\partial \lambda}{\partial \mu} \frac{\partial \lambda}{\partial \sigma} U_{\mu \sigma}^{2}}$

where $U_{\mu}$ and $U_{\sigma}$ are the uncertainties of the mean and width of the fitted Gaussian peaks and $U_{\mu \sigma}$ is the covariance of these two parameters, which are derived as part of the Levenberg-Marquardt fitting. 


\subsection{Evaluation of the success of the fitting and boundary location}

The coefficients of each fit and the estimated boundaries are checked against a number of criteria to remove auroral luminosity boundaries arising from the results of poor fitting to bad or noisy data. These criteria are based on those specified by Carbary et al. (2003) but have been adjusted and tested for use with IMAGE FUV data. The criteria used here are:

1. The Gaussian amplitude(s) $A$ must be greater than zero.

2. The Gaussian centre(s) $\mu$ must fall within the AACGM latitude range of the given intensity profile.

3. The Gaussian amplitude(s) $A$ must be at least $10 \%$ of the amplitude of the background FUV intensity at the location of the centre of that Gaussian peak (i.e., $B+C \mu+D \mu^{2}$ or $\left.E+F \mu+G \mu^{2}\right)$. In the case of the fitted double Gaussian, the amplitude of the secondary peak also must be at least $20 \%$ of the amplitude of the primary peak.

4. The Gaussian width(s) $\sigma$ must exceed the bin width of the intensity profile, i.e., $1^{\circ}$, but must not exceed the AACGM latitude range of the intensity profile.

5. The derived PALB $\lambda_{p}$ must fall within the range of the lowest latitude covered by the intensity profile and $90^{\circ}$ AACGM latitude.

Additional criteria based on measures of goodness-of-fit or uncertainty can be used to determine whether an auroral luminosity boundary estimation should be considered a success. Thresholds for $\chi^{2} / v$ or standard error can be varied to maximise either the number of estimates or to minimise the uncertainty in estimates, for example. We have used the constraint that the $\chi^{2} / v$ value must be below 10.0 for a fit to be considered successful. We also require that the uncertainty on the poleward and equatorward boundaries derived from WIC images must not exceed $1^{\circ}\left(2^{\circ}\right.$ for boundaries derived from SI12 and SI13 images). The threshold for the equatorward boundary uncertainty is included as an indication of the overall precision of the fit. When fitting a single Gaussian function, the uncertainty on the poleward and equatorward boundaries will be the same.

The $\chi^{2} / v$ statistic is also used to determine the most appropriate auroral luminosity boundary estimates when the fits of both the single Gaussian and double Gaussian function to a given latitudinal intensity profile pass all of the criteria. The fit with the lowest $\chi^{2} / v$ value is selected as the more appropriate fit to the data and the corresponding $\lambda_{\mathrm{e}}$ and $\lambda_{\mathrm{p}}$ are retained. The degrees of freedom $(v)$ of a fit to an intensity profile is simply the number of magnetic latitude bins in that profile minus the number of parameters used to specify the function for the fit. Hence, the $v$ for a single Gaussian fit to a latitudinal profile will be higher than that for a double Gaussian fit to the same profile as 6 parameters are used to specify the single Gaussian function rather than the 9 parameters of the double Gaussian function. This means that should the fits of the single and double Gaussian functions have an almost identical $\chi^{2}$ value, the fit to the single Gaussian function will be selected in preference to the fit to the double Gaussian.

In the case that one of the single and double Gaussian fits to an intensity profile passes the criteria for success detailed above while the other fails, that fit will be used to determine the PALB location. This is irrespective of which fit had the better $\chi^{2} / v$ value for that intensity profile.

\subsection{Technique limitations}

The technique as outlined requires that each MLT sector be well modelled either as a continuous single or double auroral oval and so will not provide an accurate representation of more complex auroral configurations (see Frey, 2007, for a review of localised auroral features outside the main oval). No explicit distinction is made between a double oval and other auroral features that may appear poleward of the main oval, such as high-latitude sun-aligned arcs. When present, high-latitude sun-aligned arcs can be suggestive of a configuration of multiple regions of open and closed magnetic flux rather than a single open polar cap (e.g., Newell et al., 1997; Brittnacher et al., 1999; Newell et al., 2009). For each MLT sector, only a single PALB is assumed to be a proxy for the OCB and, hence, polar caps with multiple open field line regions will not be well modelled. Our technique also assumes that the region between the main oval and a poleward bulge is on closed magnetic field lines. The technique used for automated detection of PAPBs from DMSP data also considers any void regions observed at latitudes equatorward of a closed region, such as the central plasma sheet or plasma sheet boundary layer, to also be closed (Sotirelis and Newell, 2000) and so comparison of our PALBs with this dataset is still valid. Additionally, post-processing techniques could be applied to our PALBs to identify or eliminate images showing deviation from "typical" auroral configurations, such as images with theta auroral signatures. Subauroral features, such as detached arcs and patches, may also occasionally result in inaccurate modelling of the EALB.

\section{Evaluation of the boundary location technique}

In this section, we present a case study to illustrate how our auroral luminosity boundary location technique compares with the single Gaussian model and single camera location method of Carbary et al. (2003). In addition, we present a statistical analysis of the whole dataset, in order to show more generally the advantages of modelling intensity profiles as both single and double Gaussian functions. 

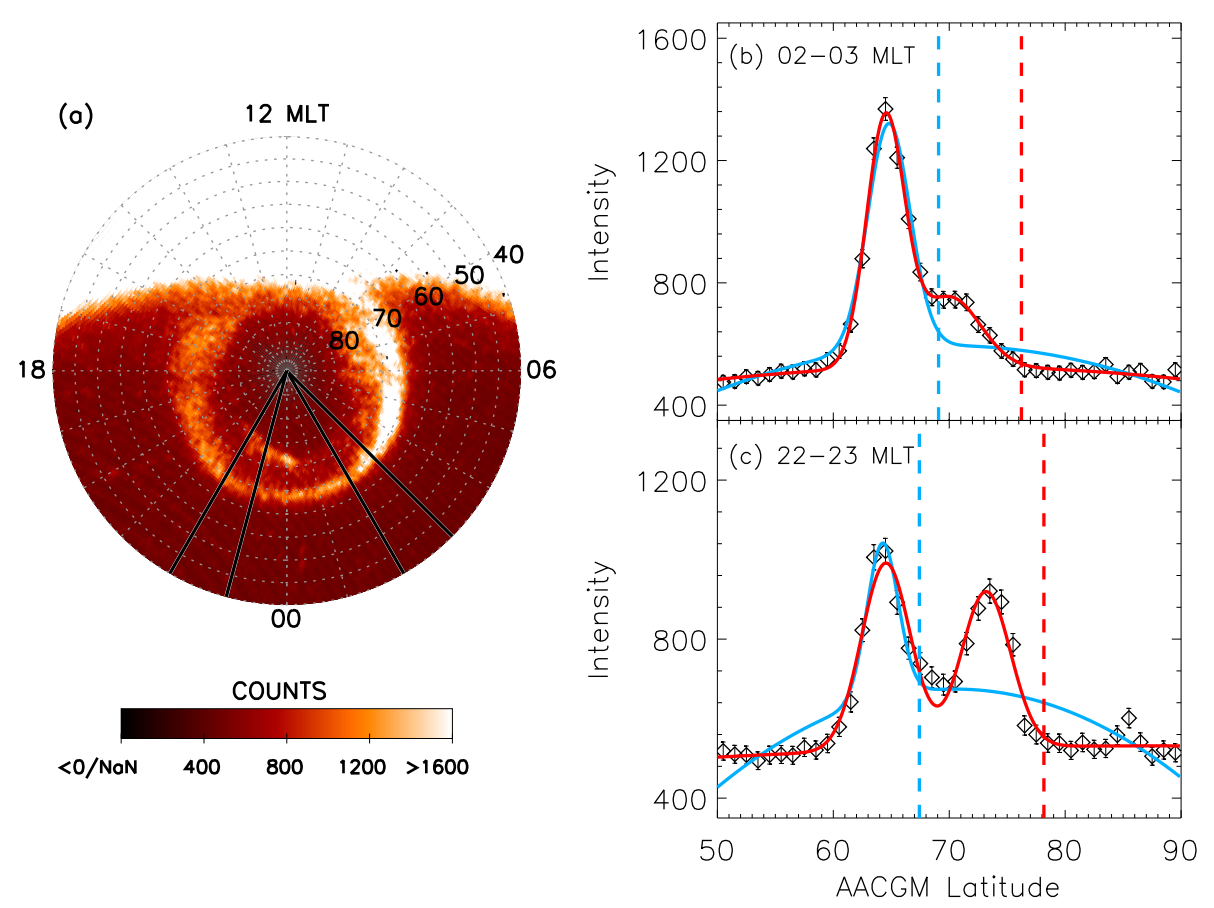

Fig. 1. FUV intensity observed by the IMAGE FUV WIC instrument at 00:10 UT 1 February 2001 in AACGM coordinates (panel a). The solid black lines mark the 02:00-03:00 and 22:00-23:00 MLT sectors. All count values above 1600 have been saturated. The latitudinal luminosity intensity profiles corresponding to these MLT sectors are shown in panels (b) and (c), respectively. The black diamonds show the mean count values of the intensity profiles. The standard error on these intensity count values are shown. The blue curves show the result of fitting the function $F_{\mathrm{S}}(\lambda)$ to these intensity profiles with the vertical blue dashed lines indicating the PALBs derived from these fits for each profile. The red curves show the result of fitting the function $F_{\mathrm{d}}(\lambda)$ to these intensity profiles with the vertical red dashed lines indicating the PALBs derived from these fits.

\subsection{Case study: boundary estimates during moderate geomagnetic activity}

Figure 1a shows the auroral luminosity variation recorded by the WIC channel of the IMAGE FUV instrument on 1 February 2001 at 00:10 UT in AACGM coordinates. Intensity values greater than 1600 counts have been saturated. The saturated values at lower magnetic latitudes across the dayside MLT sectors are caused by dayglow. This image was taken during a small geomagnetic storm driven by the passage of an interplanetary coronal mass ejection (ICME) with a minimum value of the Dst index of $-43 \mathrm{nT}$. The onset of this small storm occurred on the morning of the 31 January 2001, with minimum Dst observed at 18:00 to 19:00 UT on that day. At the time of the image, Dst was $-33 \mathrm{nT}$ while the $\mathrm{Kp}$ index had a value of 2 .

Bifurcation of auroral emission is evident in both the preand post-midnight sectors, with emission poleward of the main oval. Figure $1 \mathrm{~b}$ and $\mathrm{c}$ shows latitudinal profiles of FUV intensity (black diamonds) in the 02:00 to 03:00 MLT and 22:00 to 23:00 MLT sectors, respectively, as highlighted in Fig. 1a by the black solid lines. Error bars on the diamonds indicate the standard error of the mean intensity values. In each panel, the blue curve shows the result of fitting the sin- gle Gaussian function $F_{\mathrm{S}}(\lambda)$ to the latitudinal profile while the red curve shows the result of fitting the double Gaussian function $F_{\mathrm{d}}(\lambda)$ to that profile. The vertical dashed lines mark the location of the PALB estimated from the coefficients of each fit using Eq. (4).

The bifurcation of the auroral oval apparent in Fig. 1a is also evident in the latitudinal profiles, with emission poleward of the main peak in Fig. 1b and a largely detached secondary peak in Fig. 1c. In both cases, the double Gaussian fit provides a better description of the underlying intensity profile and was selected in preference to the single Gaussian fit; in the 02:00 to 03:00 MLT sector, $\chi^{2} / v$ for the $F_{\mathrm{d}}(\lambda)$ fit is $\sim 0.4$ compared to $\sim 4.9$ for the $F_{\mathrm{s}}(\lambda)$ fit, and in the 22:00 to 23:00 MLT sector, the $\chi^{2} / \nu$ for the $F_{\mathrm{d}}(\lambda)$ fit is $\sim 1.7$ compared to $\sim 11.1$ for the $F_{\mathrm{S}}(\lambda)$ fit. It is also clear that the quadratic coefficients of the single Gaussian function are poorly fit to the background emissions of the intensity profile due to the presence of the second peak in auroral emission in the 22:00 to 23:00 MLT sector.

The PALBs estimated from the function $F_{\mathrm{d}}(\lambda)$ are located poleward of both intensity peaks for both profiles and appear to correspond to a return to background FUV intensity levels poleward of the auroral oval. They also pass our criteria for successful boundary location. In the 02:00 to 03:00 MLT 

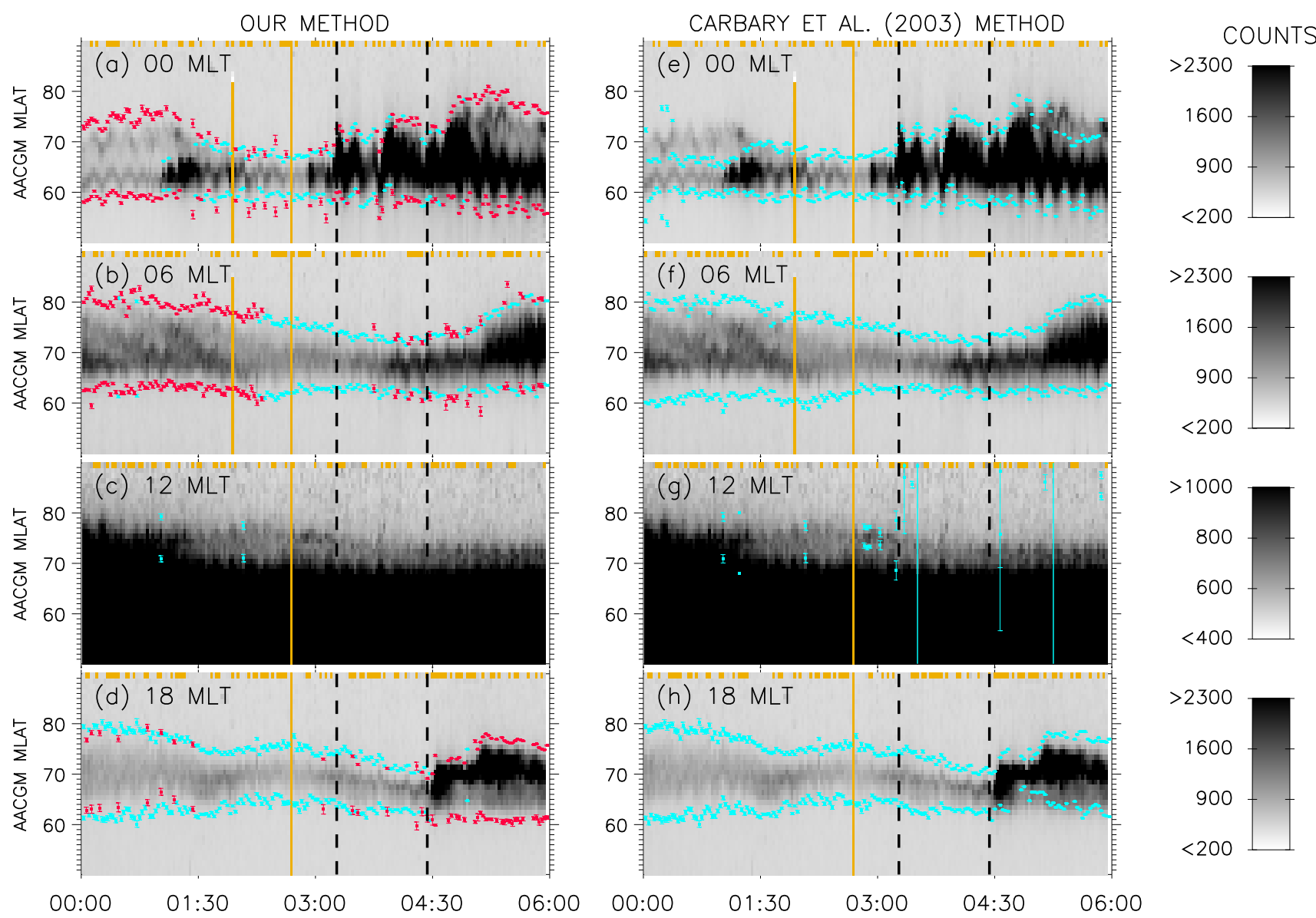

Fig. 2. Intensity counts recorded by the IMAGE FUV WIC sensor on the 1 February 2001 between 00:00 and 06:00 UT. Count values above and below thresholds indicated in the intensity scales have been saturated. Not a Number $(\mathrm{NaN})$ and zero count intensity values are shown in yellow. From top to bottom, the panels show the MLT sectors 00:00-01:00, 06:00-07:00, 12:00-13:00, and 18:00-19:00, respectively. Panels (a) to (d) show auroral luminosity boundary locations derived according to the method detailed in this paper. Blue squares denote poleward and equatorward luminosity boundaries determined from fits to a function with a single Gaussian component $\left(F_{\mathrm{S}}(\lambda)\right)$, with blue error bars showing $\pm U_{\lambda}$ on these boundaries. Red squares denote poleward and equatorward luminosity boundaries determined from fits to a function with a double Gaussian component $\left(F_{\mathrm{d}}(\lambda)\right.$ ), with red error bars showing $\pm U_{\lambda}$ on these boundaries. Panels e to h show these data with auroral luminosity boundary locations derived according to the method detailed in Carbary et al. (2003). Vertical dashed black lines indicate times of substorm onset from the list outlined in Frey et al. (2004).

sector (Fig. 1b), the single Gaussian fit also passes the criteria for success, which could have resulted in an incorrectly estimated PALB. However, the single Gaussian fit in the 22:00 to 23:00 MLT sector failed the criteria and would have been discarded.

Figure 2 shows keograms of auroral luminosity intensity in four MLT sectors recorded by WIC from 00:00 to 06:00 UT 1 February 2001. In this time period, Dst was in the range $-29 \mathrm{nT}$ to $-36 \mathrm{nT}$ while $\mathrm{Kp}$ was in the range 2 to $3^{+}$. In this figure, auroral luminosity boundaries derived from fits to the function $F_{\mathrm{S}}(\lambda)$ are indicated as blue squares and those derived from fits to the function $F_{\mathrm{d}}(\lambda)$ are indicated as red squares. Error bars show the upper and lower uncertainty bounds on these boundary estimates $\left(U_{\lambda}\right)$. Panels on the left hand side of the figure show the auroral luminosity boundary locations derived using the technique outlined in this paper while the right hand side panels show those derived using the technique of Carbary et al. (2003). We include the boundaries obtained using the method of Carbary et al. (2003) to enable direct comparison of our method with another fully automated boundary location technique that only employs fits to a single Gaussian function when deriving PALB locations. These PALBs have not been corrected for their systematic offset with DMSP PAPBs, and hence the boundary locations derived using the method outlined in this paper from a single Gaussian function, $F_{\mathrm{s}}(\lambda)$, differ from those derived using the method of Carbary et al. (2003) only by their different success criteria. The list of substorm onsets detected from IMAGE data in Frey et al. (2004) includes two onsets during the period shown in Fig. 2. These occurred at $\sim 03: 16$ UT 
and $~ 04: 26$ UT and are highlighted by the vertical dashed black lines in the figure. Oscillations in the latitude of auroral emissions, and, consequently PALB locations, with a period of around 10 to $20 \mathrm{~min}$ are evident in the keograms, and are particularly clear in the 00:00 MLT sector prior to 01:00 UT. These oscillations are caused by inconsistency in the IMAGE pointing calculation due to spin-axis coning of the satellite (as discussed earlier). Other contemporaneous images of the aurora, such as those obtained from the NORSTAR $630 \mathrm{~nm}$ meridian scanning photometer (not shown) do not show this oscillation.

At the start of the interval, prior to $\sim 01: 30 \mathrm{UT}$, bifurcation of auroral emission is evident in the 00:00 to 01:00 MLT sector. The PALBs estimated from single Gaussian fits (Fig. 2e) typically correspond to the more equatorward emission band and appear to be at erroneously low latitudes. The PALBs estimated from double Gaussian fits (Fig. 2a) appear to cover the full extent of auroral emission during this time. For a very small number of intensity profiles, auroral luminosity boundary estimates have been made from the single Gaussian fits in preference to double Gaussian fits. In these cases, the relatively low amplitude of the secondary emission with respect to the main oval causes the double Gaussian fit to fail the criteria set out in Sect. 3.2 (specifically, criterion 3) while the single Gaussian fits pass all of our criteria, hence the auroral boundaries are taken from the fits to $F_{\mathrm{S}}(\lambda)$. Bifurcation is also evident in the 00:00 to 01:00 and 18:00 to 19:00 MLT sectors following the onset of substorm activity. At these times, the double Gaussian appears to be a more appropriate model for the auroral emissions. Prior to $\sim 02: 17$ UT, the complex auroral emission profiles observed in the 06:00 to 07:00 MLT sector also appear to be better fit by double Gaussian function than a single Gaussian function from comparison of the boundaries shown in Fig. $2 b$ and f, especially the EALBs. Panels (c) and (g) of Fig. 2 show the limited number of auroral luminosity boundary locations that have been estimated in the 12:00 to 13:00 MLT sector, because dayglow contamination swamps the auroral emission.

In the 18:00 to 19:00 MLT sector (Fig. 2d), it can be seen that some of the auroral boundaries prior to $\sim 01: 30 \mathrm{UT}$ and again between $\sim 03: 00$ UT and 04:30 UT switch between those estimated from a single Gaussian function and those estimated from a double Gaussian function. This switching appears to be caused by fluctuations in the amplitudes of two closely-spaced Gaussian components in the auroral emission profiles such that at times the two peaks cannot be resolved and appear as one. At the transition between an apparently single and a resolved bifurcated oval, intensity profiles are not clearly better modelled as either $F_{\mathrm{s}}(\lambda)$ or $F_{\mathrm{d}}(\lambda)$, resulting in the switching behaviour.

To illustrate the difference between the IMAGE FUV cameras, Fig. 3 shows the mean intensity counts recorded by the SI12 (panels a to d) and SI13 (panels e to h) channels during the same moderate storm period with auroral luminosity boundary locations estimated using the method outlined in
Sect. 3. As before, blue squares indicate boundaries derived from single Gaussian fits to the intensity profiles while red squares indicate boundaries derived from double Gaussian fits. In contrast to the WIC data shown in Fig. 2, bifurcation is not evident in the SI12 auroral emissions and consequently fewer boundaries are made from double Gaussian fits. In particular, the more poleward band of emission observed prior to $\sim 01: 30$ UT in the WIC emissions in the 00:00 to 01:00 MLT sector (Fig. 2a) is not obvious in the SI12 emissions (Fig. 3a). Some dayside boundaries have been resolved from the SI12 auroral emission due to the reduced dayglow contamination at this wavelength. However, the SI13 auroral emissions show some bifurcation in the 00:00 to 01:00 MLT sector at the start of the period and following the second substorm onset (Fig. 3e) but does not capture the bifurcation evident in the WIC auroral emissions in the dawn and dusk sectors. This is likely the result of the lower resolution of the SI13 camera relative to the separation of the peaks in auroral intensity.

\subsection{Statistical analysis of the impact of the double Gaussian model on boundary estimation}

The number of PALB locations successfully estimated from images from each of the three FUV detectors between May 2000 and August 2002 is shown in Fig. 4a. As can been seen, the lowest numbers of successful PALB locations occur in dayside MLTs for all three cameras. This is due to a combination of the effects of dayglow and weak dayside auroral emissions. Peak numbers of successful PALB locations from SI12 and SI13 images occur around local midnight, while peak successful PALB locations from WIC images occur around dawn and dusk MLTs. The primary cause of failure in PALB estimation in all MLT sectors for intensity profiles from SI12 and SI13 images is the uncertainty in boundary location exceeding the acceptable limit specified in Sect. 3.2. Additionally, a large number of double Gaussian fits fail our width criteria (criterion 4). In nightside MLT sectors, there are fewer successful PALBs derived from WIC images than may be expected from the trends in SI12 and SI13 PALB numbers. This is a largely seasonal effect, with few "nightside" boundaries being successfully located during summer months from WIC images due to dayglow contamination beyond the geomagnetic pole.

Figure $4 \mathrm{~b}$ shows the percentage of those successfully estimated auroral luminosity boundary locations made from a fit of $F_{\mathrm{d}}(\lambda)$ to a latitudinal intensity profile in preference to $F_{\mathrm{S}}(\lambda)$. A clear MLT dependence is evident, with the highest percentages observed around local midnight. WIC images have higher percentages of intensity profiles that are better modelled as a double Gaussian function than images from SI12 and SI13 in each MLT sector, with 35\% of WIC profiles in the 23:00 to 01:00 MLT sector being better modelled as a double Gaussian. The difference between the SI13 and WIC imagers, which are both sensitive to electron emissions, 


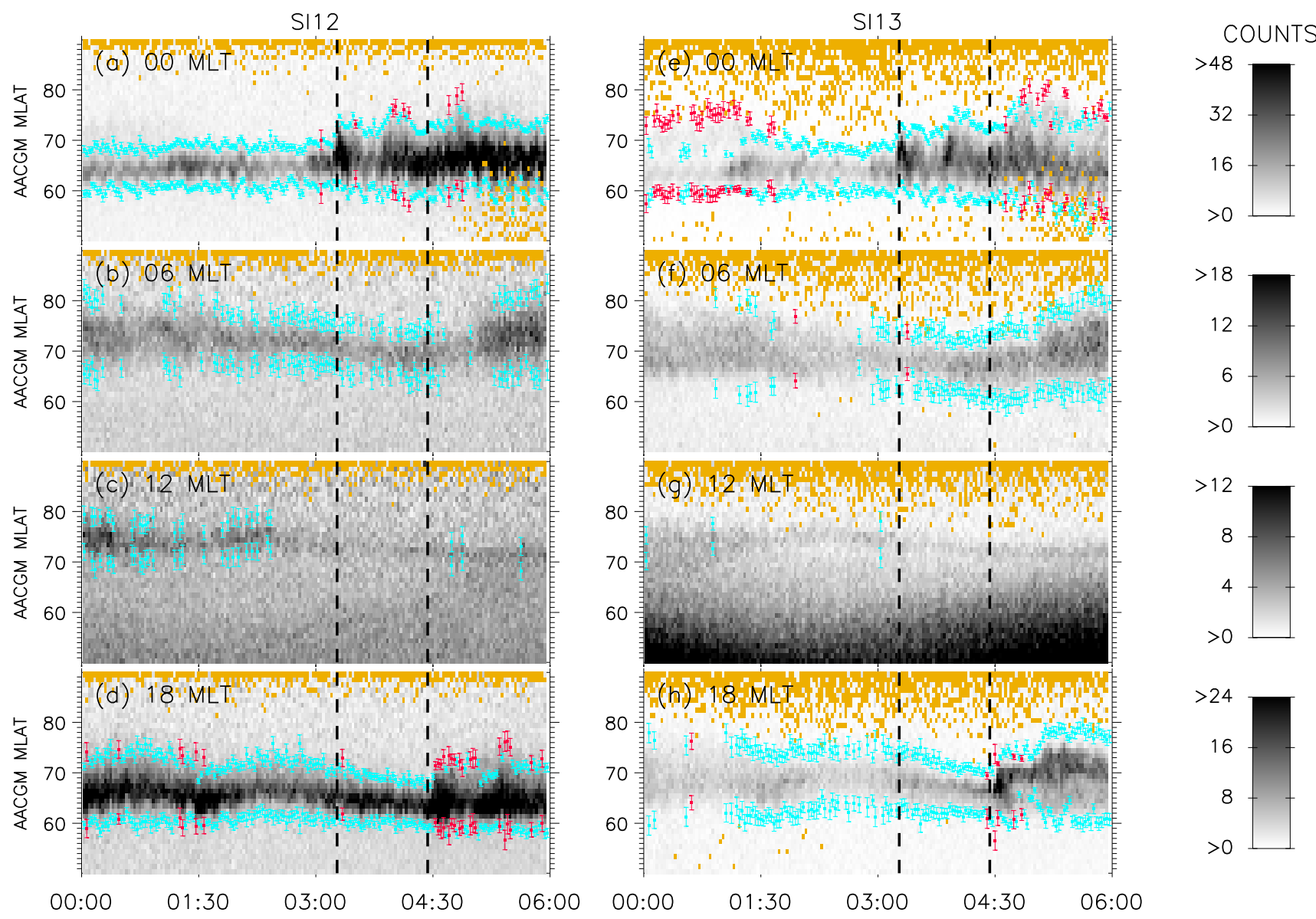

Fig. 3. Intensity counts recorded by the IMAGE FUV SI12 and SI13 sensors on the 1 February 2001 between 00:00 and 06:00 UT. Count values above and below thresholds indicated in the intensity scales have been saturated. Not a Number (NaN) and zero count intensity values are shown in yellow. From top to bottom, the panels show the MLT sectors 00:00-01:00, 06:00-07:00, 12:00-13:00 and 18:0019:00, respectively. Panels (a) to (d) show auroral luminosity boundary locations derived according to the method detailed in this paper from SI12 images, with panels (e) to (h) showing the same derived from SI13 images. Blue squares denote poleward and equatorward luminosity boundaries determined from fits to a function with a single Gaussian component $\left(F_{\mathrm{S}}(\lambda)\right.$ ), with blue error bars showing $\pm U_{\lambda}$ on these boundaries. Red squares denote poleward and equatorward luminosity boundaries determined from fits to a function with a double Gaussian component $\left(F_{\mathrm{d}}(\lambda)\right)$, with red error bars showing $\pm U_{\lambda}$ on these boundaries. Vertical dashed black lines indicate times of substorm onset from the list outlined in Frey et al. (2004).

is likely due to the lower imager resolution and lower typical intensity count levels of SI13, making the bifurcation of auroral emission more difficult to resolve in SI13 images. Similarly, this is also the case for SI12 but with the additional differences due to proton rather than electron emissions.

Table 1 compares the number of successful PALB locations made using our technique (showing both those boundaries made from fits of the single Gaussian function $\left(F_{\mathrm{s}}(\lambda)\right)$ and those made from fits of the double Gaussian function $\left(F_{\mathrm{d}}(\lambda)\right)$ ), and using the technique outlined in Carbary et al. (2003) (WIC only). It also shows how these numbers vary with geomagnetic activity (as measured by Kp) and MLT (6-h sectors). Consistent with Fig. 4, PALBs made from fits of $F_{\mathrm{d}}(\lambda)$ using our technique are most numerous in the nightside MLT sectors (21:00 to 03:00 MLT) and least numerous in the dayside (09:00 to 15:00 MLT). Additionally, the percentage of the total number of our successfully located PALBs being made from fits of $F_{\mathrm{d}}(\lambda)$ in preference to $F_{\mathrm{s}}(\lambda)$ in the nightside increases with geomagnetic activity, with more than $50 \%$ of nightside boundaries made from WIC images resulting from double Gaussian fits. Additionally, the percentage of successfully located PALBs from the available luminosity profiles also increases with geomagnetic activity in all MLT ranges for each of the FUV detectors (with the exception of WIC in nightside sector where similar percentages of PALBs are successfully located during moderate and active conditions). From comparison with the numbers of PALB locations successfully estimated using the technique 

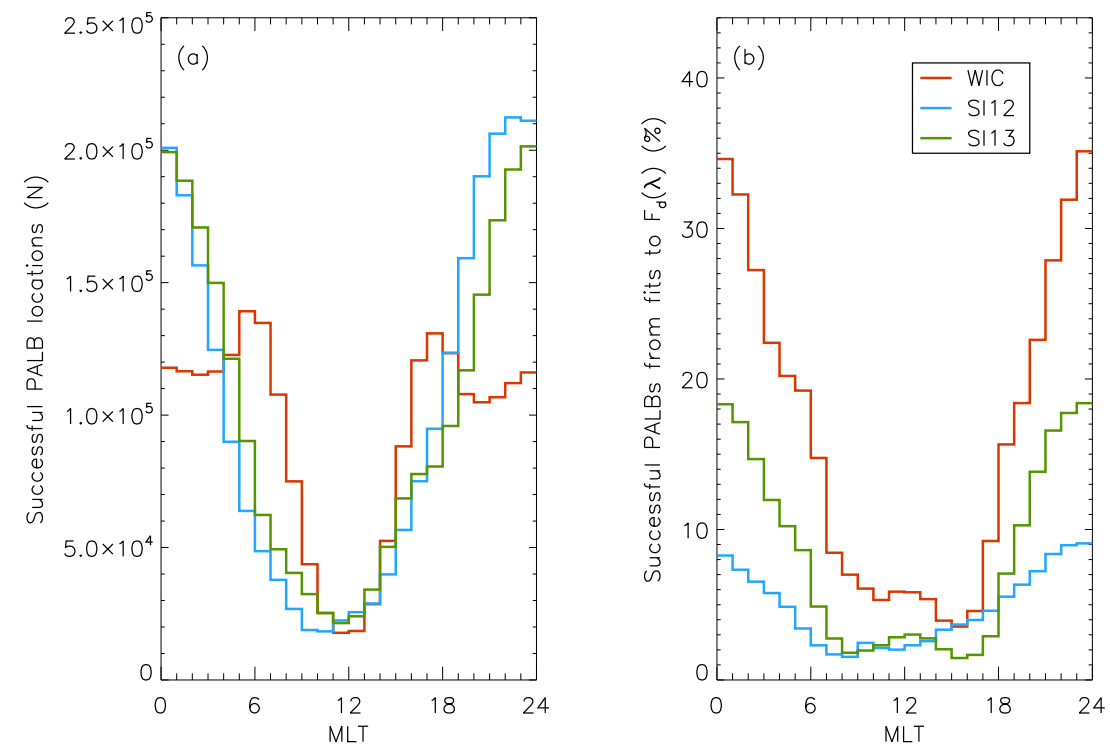

Fig. 4. Number of PALBs in each MLT sector derived from IMAGE FUV WIC images (red line), SI12 images (blue line), and SI13 images (green line) from May 2000 until August 2002 using the technique outlined in this paper (panel a). Panel (b) shows the percentage of these successful boundary locations that are derived from fits of the function $F_{\mathrm{d}}(\lambda)$ to latitudinal intensity profiles.

Table 1. Number $(N)$ of PALB estimations derived from IMAGE FUV images between May 2000 and August 2002 using the method outlined in this paper (all three FUV detectors) and the method of Carbary et al. (2003) (WIC images only) separated by MLT and geomagnetic activity. The percentages of successfully located PALBs out of the total available luminosity profiles are shown in parentheses. The percentages of successfully located PALBs made from single Gaussian fits $\left(F_{\mathrm{S}}(\lambda)\right)$ and double Gaussian fits $\left(F_{\mathrm{d}}(\lambda)\right)$ are also shown.

\begin{tabular}{|c|c|c|c|c|c|c|c|c|c|c|}
\hline \multirow[b]{2}{*}{ MLT } & \multicolumn{3}{|c|}{ SI12 } & \multicolumn{3}{|c|}{$\begin{array}{l}\text { Our method } \\
\text { SI13 }\end{array}$} & \multicolumn{3}{|c|}{ WIC } & \multirow{2}{*}{$\begin{array}{c}\text { Carbary method } \\
\text { WIC } \\
\text { N }\end{array}$} \\
\hline & $N$ & $F_{\mathrm{S}}(\lambda)$ & $F_{\mathrm{d}}(\lambda)$ & $\mathrm{N}$ & $F_{\mathrm{S}}(\lambda)$ & $F_{\mathrm{d}}(\lambda)$ & $\mathrm{N}$ & $F_{\mathrm{S}}(\lambda)$ & $F_{\mathrm{d}}(\lambda)$ & \\
\hline 21:00-03:00 & $392182(35 \%)$ & $96 \%$ & $4 \%$ & $404304(37 \%)$ & $91 \%$ & $9 \%$ & $273536(27 \%)$ & $81 \%$ & $19 \%$ & $371707(36 \%)$ \\
\hline 03:00-09:00 & $85264(8 \%)$ & $98 \%$ & $2 \%$ & $143935(13 \%)$ & $94 \%$ & $6 \%$ & $217696(21 \%)$ & $87 \%$ & $13 \%$ & $254310(25 \%)$ \\
\hline 09:00-15:00 & $24919(2 \%)$ & $98 \%$ & $2 \%$ & $59808(6 \%)$ & $98 \%$ & $2 \%$ & $59722(6 \%)$ & $94 \%$ & $6 \%$ & $61253(6 \%)$ \\
\hline $15: 00-21: 00$ & $164039(15 \%)$ & $98 \%$ & $2 \%$ & $165651(15 \%)$ & $98 \%$ & $2 \%$ & $198802(19 \%)$ & $94 \%$ & $6 \%$ & $217504(21 \%)$ \\
\hline \multicolumn{11}{|c|}{ During moderate activity $\quad 2 \leq \mathrm{Kp}<4$} \\
\hline 21:00-03:00 & $595584(60 \%)$ & $91 \%$ & $9 \%$ & $548541(56 \%)$ & $81 \%$ & $19 \%$ & $327845(35 \%)$ & $63 \%$ & $37 \%$ & $391158(41 \%)$ \\
\hline 03:00-09:00 & $206120(21 \%)$ & $97 \%$ & $3 \%$ & $263827(27 \%)$ & $92 \%$ & $8 \%$ & $360710(38 \%)$ & $84 \%$ & $16 \%$ & $361173(38 \%)$ \\
\hline 09:00-15:00 & $77375(8 \%)$ & $98 \%$ & $2 \%$ & $94069(10 \%)$ & $98 \%$ & $2 \%$ & $90003(10 \%)$ & $95 \%$ & $5 \%$ & $77393(8 \%)$ \\
\hline $15: 00-21: 00$ & $384581(39 \%)$ & $95 \%$ & $5 \%$ & $313221(32 \%)$ & $94 \%$ & $6 \%$ & $369010(39 \%)$ & $88 \%$ & $12 \%$ & $359926(38 \%)$ \\
\hline \multicolumn{11}{|c|}{ During high activity $\mathrm{Kp} \geq 4$} \\
\hline 21:00-03:00 & $182071(71 \%)$ & $85 \%$ & $15 \%$ & $172958(69 \%)$ & $71 \%$ & $29 \%$ & $82717(34 \%)$ & $49 \%$ & $51 \%$ & $96697(40 \%)$ \\
\hline 03:00-09:00 & $100193(39 \%)$ & $92 \%$ & $8 \%$ & $105465(42 \%)$ & $86 \%$ & $14 \%$ & $117050(48 \%)$ & $78 \%$ & $22 \%$ & $113837(47 \%)$ \\
\hline 09:00-15:00 & $51700(20 \%)$ & $96 \%$ & $4 \%$ & $33588(13 \%)$ & $97 \%$ & $3 \%$ & $36658(15 \%)$ & $95 \%$ & $5 \%$ & $23686(10 \%)$ \\
\hline $15: 00-21: 00$ & $150736(59 \%)$ & $89 \%$ & $11 \%$ & $106082(42 \%)$ & $81 \%$ & $19 \%$ & $107752(45 \%)$ & $75 \%$ & $25 \%$ & $102009(42 \%)$ \\
\hline
\end{tabular}

of Carbary et al. (2003), it can be seen that the requirement of our technique to discriminate between single and double Gaussian models of auroral luminosity does not greatly reduce the number of boundary locations found $(<10 \%)$.
Figure 5 illustrates the effect of modelling the latitudinal intensity profiles as a double Gaussian function on the estimated auroral luminosity boundary locations. It shows the distribution of differences between boundaries estimated 


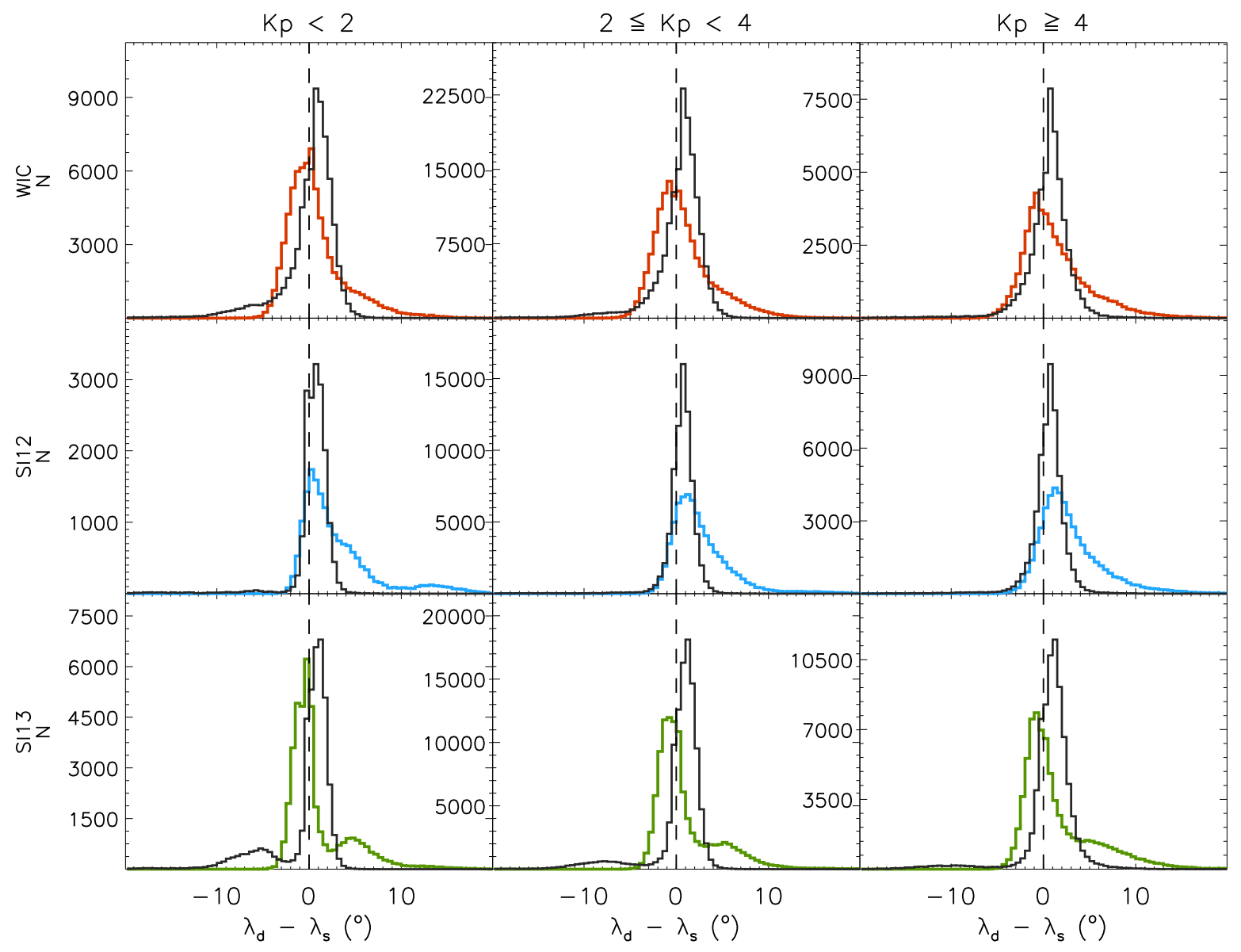

Fig. 5. Distribution of differences in auroral luminosity boundaries derived from single Gaussian $\left(F_{\mathrm{S}}(\lambda)\right)$ and double Gaussian fits $\left(F_{\mathrm{d}}(\lambda)\right)$ when both models successfully produce boundary locations under differing levels of geomagnetic activity, inferred from the Kp index. The coloured curves show the distribution of PALB differences for each of the three IMAGE FUV detectors during May 2000 to August 2002. The black curves in each panel show the distribution of EALB differences.

Table 2. Sample size $(N)$, mean $(\mu)$, standard deviation $(\sigma), 25$ th percentile $(25 \%)$, median $(50 \%)$, and 75 th percentile $(75 \%)$ for the differences between DMSP PAPBs and IMAGE FUV PALBs, during 2000 and 2001. Differences have been included only when PALBs are successfully located from fits of both single and double Gaussian functions to an auroral intensity profile and the double Gaussian is the superior model.

\begin{tabular}{l|r|rrrrr|rrrrr}
\hline & & \multicolumn{5}{|c|}{$F_{\mathrm{S}}(\lambda)$} & \multicolumn{5}{c}{$F_{\mathrm{d}}(\lambda)$} \\
& $N$ & $\mu$ & $\sigma$ & $25 \%$ & $50 \%$ & $75 \%$ & $\mu$ & $\sigma$ & $25 \%$ & $50 \%$ & $75 \%$ \\
\hline WIC & 370 & -1.67 & 3.60 & -3.55 & -1.88 & -0.02 & -1.94 & 3.18 & -3.02 & -1.74 & -0.49 \\
SI12 & 140 & 1.42 & 3.44 & -0.93 & 1.40 & 3.70 & -1.17 & 3.08 & -2.69 & -1.48 & 0.57 \\
SI13 & 219 & -0.98 & 5.30 & -3.24 & -1.28 & 0.96 & -1.30 & 4.58 & -2.15 & -1.07 & 0.17 \\
\hline
\end{tabular}

from fits of the single Gaussian function $\left(F_{\mathrm{s}}(\lambda)\right)$ and the double Gaussian function $\left(F_{\mathrm{d}}(\lambda)\right)$ when both models produce successful boundary locations. In nearly all cases (>99\%), the double Gaussian function is a better model than the single Gaussian function. The coloured curves show the distribution of differences in the derived PALBs while the black curves show the differences in EALBs. The boundary diffe- rences have been separated according to geomagnetic activity and FUV camera. Each distribution of PALB differences shows a main narrow peak with a modal value close to zero and a secondary peak or heavy skew for large, positive differences. These distributions imply two classes of offset between PALBs estimated from $F_{\mathrm{s}}(\lambda)$ and $F_{\mathrm{d}}(\lambda)$; small offsets that do not show a significant poleward or equatorward bias 
Table 3. The number $(\mathrm{N})$ and mode $(\mathrm{M})$ of comparisons of differences between DMSP PAPBs and FUV PALBs during 2000 and 2001 and inter-camera comparisons from May 2000 to August 2002. All available boundaries have been included. Estimated values for modal differences are shown in square brackets.

\begin{tabular}{|c|c|c|c|c|c|c|c|c|c|c|c|c|}
\hline \multirow[b]{2}{*}{ MLT } & \multicolumn{2}{|c|}{$\lambda_{\mathrm{DMSP}}-\lambda_{\mathrm{WIC}}$} & \multicolumn{2}{|c|}{$\lambda_{\mathrm{DMSP}}-\lambda_{\mathrm{SI} 12}$} & \multicolumn{2}{|c|}{$\lambda_{\mathrm{DMSP}}-\lambda_{\mathrm{SI} 13}$} & \multicolumn{2}{|c|}{$\lambda_{\mathrm{WIC}}-\lambda_{\mathrm{SI} 12}$} & \multicolumn{2}{|c|}{$\lambda_{\mathrm{WIC}}-\lambda_{\mathrm{SI} 13}$} & \multicolumn{2}{|c|}{$\lambda_{\mathrm{SI} 12}-\lambda_{\mathrm{SI} 13}$} \\
\hline & $\mathrm{N}$ & M & $\mathrm{N}$ & M & $\mathrm{N}$ & M & $\mathrm{N}$ & $\mathrm{M}$ & $\mathrm{N}$ & M & $\mathrm{N}$ & $\mathrm{M}$ \\
\hline 00:00-01:00 & - & {$[0.0]$} & - & {$[-1.5]$} & - & {$[-1.0]$} & 88096 & 0.2 & 99123 & 0.0 & 149128 & 0.0 \\
\hline 01:00-02:00 & - & {$[0.0]$} & - & {$[-1.5]$} & - & {$[-0.5]$} & 82105 & -0.2 & 95789 & -0.2 & 135371 & 0.2 \\
\hline $02: 00-03: 00$ & - & {$[0.5]$} & - & {$[-1.5]$} & - & {$[0.0]$} & 72059 & -0.4 & 89998 & 0.0 & 113759 & 0.4 \\
\hline 03:00-04:00 & - & {$[0.0]$} & - & {$[-1.0]$} & - & {$[0.0]$} & 60409 & -0.8 & 83852 & -0.4 & 89959 & 0.8 \\
\hline 04:00-05:00 & - & {$[0.0]$} & - & {$[-0.5]$} & - & {$[0.0]$} & 49082 & -0.8 & 76231 & -0.4 & 62989 & 1.2 \\
\hline 05:00-06:00 & 143 & -0.5 & 32 & -1.5 & 48 & 0.5 & 40537 & -0.6 & 64875 & 0.2 & 40031 & 0.8 \\
\hline 06:00-07:00 & 570 & -0.5 & 191 & 0.0 & 245 & -0.5 & 30840 & -0.4 & 45323 & -0.4 & 23026 & 1.0 \\
\hline 07:00-08:00 & 281 & -1.5 & 107 & 0.0 & 144 & -1.0 & 20696 & 0.6 & 32757 & 0.0 & 14668 & 1.0 \\
\hline 08:00-09:00 & 221 & -2.0 & 74 & -1.5 & 120 & -1.0 & 11874 & 0.2 & 20908 & 0.0 & 8994 & 0.4 \\
\hline 09:00-10:00 & 174 & -2.0 & 66 & -2.0 & 159 & -1.0 & 6545 & 0.0 & 11596 & -0.2 & 5737 & 0.4 \\
\hline $10: 00-11: 00$ & 55 & -1.0 & 47 & -2.5 & 79 & -1.5 & 4655 & 0.4 & 6409 & 0.4 & 5453 & 0.4 \\
\hline $11: 00-12: 00$ & 38 & -2.0 & 40 & -3.5 & 48 & -1.5 & 4107 & 0.4 & 4309 & 0.2 & 5777 & 1.0 \\
\hline $12: 00-13: 00$ & 20 & -1.5 & 31 & -2.0 & 22 & -2.0 & 4184 & -0.2 & 4881 & 0.4 & 5947 & 0.4 \\
\hline $13: 00-14: 00$ & 36 & -1.5 & 22 & -1.5 & 38 & -1.5 & 5559 & -0.4 & 8023 & 0.2 & 6887 & 0.4 \\
\hline $14: 00-15: 00$ & 70 & -1.5 & 41 & -1.0 & 68 & -1.5 & 10350 & -0.2 & 16748 & 0.2 & 10225 & 0.0 \\
\hline $15: 00-16: 00$ & 156 & -1.5 & 99 & 0.0 & 137 & -1.0 & 20845 & 0.6 & 33238 & 0.4 & 18048 & 0.0 \\
\hline $16: 00-17: 00$ & 490 & -1.5 & 281 & 0.5 & 277 & -1.0 & 37503 & 0.8 & 49486 & 0.4 & 28013 & -0.4 \\
\hline $17: 00-18: 00$ & 793 & -1.5 & 571 & 0.0 & 533 & -1.0 & 51964 & 1.8 & 55185 & 0.2 & 38659 & -1.0 \\
\hline 18:00-19:00 & 551 & -2.0 & 550 & 1.0 & 478 & -1.0 & 63210 & 1.8 & 63985 & 0.2 & 58179 & -0.8 \\
\hline $19: 00-20: 00$ & 282 & -1.5 & 425 & 1.0 & 328 & -1.5 & 66233 & 2.0 & 69336 & 0.2 & 81368 & -1.0 \\
\hline $20: 00-21: 00$ & 357 & -1.5 & 541 & 1.0 & 482 & -1.5 & 72958 & 1.6 & 76338 & 0.0 & 109780 & -1.0 \\
\hline $21: 00-22: 00$ & 206 & -1.5 & 379 & 0.0 & 326 & -1.5 & 79412 & 1.0 & 84445 & 0.2 & 134310 & -0.8 \\
\hline $22: 00-23: 00$ & - & {$[-1.0]$} & - & {$[-0.5]$} & - & {$[-1.5]$} & 85084 & 0.8 & 92218 & 0.0 & 148770 & -0.2 \\
\hline 23:00-00:00 & - & {$[-0.5]$} & - & {$[-1.0]$} & - & {$[-1.0]$} & 88710 & 0.6 & 97808 & 0.0 & 154217 & -0.2 \\
\hline Total & 4443 & - & 3497 & - & 3532 & - & 1057017 & - & 1282861 & - & 1449295 & - \\
\hline
\end{tabular}

and large offsets where the PALB from $F_{\mathrm{d}}(\lambda)$ is consistently poleward of the PALB from $F_{\mathrm{S}}(\lambda)$. This trend is present for all levels of geomagnetic activity. The narrow peak of the distribution located close to zero is likely to be from times where the auroral emission luminosity profiles exhibit two closely located or overlapping peaks. The large differences are likely to be from times when there is clear separation in the auroral emissions, with the PALBs from double Gaussian fits consistently being made from the more poleward peak while the PALB from the single Gaussian fit is made from the more equatorward peak. The EALB difference distributions are approximately anti-symmetric to the PALB ones, particularly for boundaries from WIC and SI13 during times of low geomagnetic activity. This demonstrates that when the single and double Gaussian models produce very different auroral luminosity boundaries, the poleward (equatorward) boundaries of the double Gaussian model are consistently poleward (equatorward) of those from the single Gaussian models.

Figure 6 shows the probability densities of PALB locations for each of the three IMAGE FUV detectors separated into 6$\mathrm{h}$ MLT ranges. In each panel, the solid coloured bars show the densities for PALBs estimated from double Gaussian fits while the black curves show the densities for PALBs estimated from single Gaussian fits. In the dusk sector (15:00 to 21:00 MLT), the centroids of the density distributions of PALBs from single Gaussian fits are slightly poleward of the centroids of the density distributions of PALBs from double Gaussian fits from WIC and SI13. In all other MLT sectors and for SI12 boundaries, the PALBs centroids of the density distributions of the PALBs from double Gaussian fits are poleward of those relating to single Gaussian fits. In most cases, this suggests that the auroral luminosity boundaries are poleward of the main peak in emission. However, the distribution of PALBs from double Gaussian fits to WIC profiles in the dayside MLT sector indicates some potentially erroneous boundaries due to the relatively high probability of boundaries being located close to the cutoff of $90^{\circ}$ compared to the other MLT sectors and instruments. While these boundaries could indicate the presence of auroral features at high latitudes, they do not appear in the distributions for SI12 and SI13 cameras, which are less susceptible to dayglow contamination. 


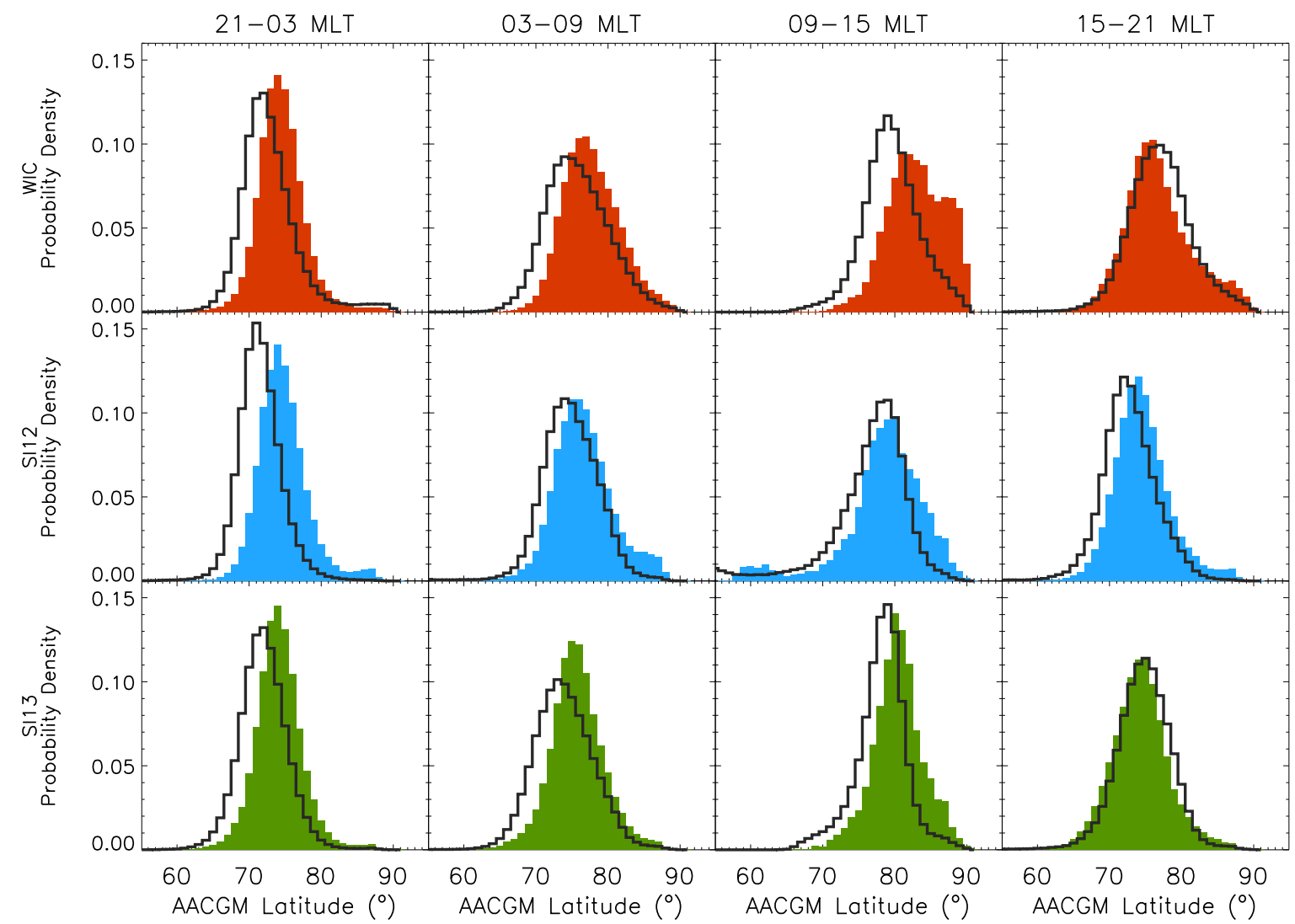

Fig. 6. Probability densities of IMAGE FUV PALBs derived using the method outlined in this paper during May 2000 to August 2002 with a resolution of $1^{\circ}$. From top to bottom, panels show the densities for PALBs from the WIC, SI12 and SI13 cameras, respectively. From left to right, the panels show the probability densities for nightside, dawnside, dayside, and duskside MLT sectors, respectively. The solid coloured bars show the densities for PALBs derived from double Gaussian $\left(F_{\mathrm{d}}(\lambda)\right)$ fits to intensity profiles while the black curves show the densities for those derived from single Gaussian $\left(F_{\mathrm{S}}(\lambda)\right)$ fits to intensity profiles. All successfully located PALBs are included.

\subsection{Statistical comparison of FUV PALBs with DMSP PAPBs}

As with similar studies (e.g. Carbary et al., 2003; Boakes et al., 2008), we compare our estimated PALBs with contemporaneous, co-located DMSP PAPBs (where available) to assess their accuracy as a proxy for the OCB. Boundary comparisons are made to the closest successful PALB within \pm 5 min UT and within $\pm 0.5 \mathrm{~h}$ MLT of the DMSP PAPB during the period from May 2000 until December 2001. The distributions of the differences between DMSP PAPBs and IMAGE PALBs across all available MLT sectors are shown in Fig. 7a to c, with a resolution of $0.5^{\circ}$. Consistent with the study of Carbary et al. (2003), these boundary difference distributions are roughly Gaussian in profile, although the SI12 PALB distribution exhibits a positive skew, indicating a higher proportion of events where the DMSP PAPB is located poleward of our FUV PALB. This is similar to the observation in the earlier case study of SI12 PALBs being made from an equatorward band of auroral emission in the 00:00 to 01:00 MLT sector between around 00:00 to 01:30 UT while
WIC and SI13 PALBs are made from a clear poleward band of emission (Figs. 2a, 3a, and 3e). The pointing calculation error for the IMAGE dataset and subsequent effect on the accuracy of the derived PALBs may increase the uncertainty of these PALBs in comparison with the PAPBs. Panels (d) to (f) of Fig. 7 illustrate the effect of fitting a double Gaussian function $\left(F_{\mathrm{d}}(\lambda)\right)$ on these boundary differences. In these panels, the solid bars show the distribution of differences between DMSP PAPBs and IMAGE PALBs derived from fits of $F_{\mathrm{d}}(\lambda)$ to intensity profiles when the fits from this function were superior to those of fits to the single Gaussian function $F_{\mathrm{S}}(\lambda)$. We only include boundaries when the PALB derived from the single Gaussian fit have also passed our criteria. The distributions of differences between PAPBs and PALBs estimates from these single Gaussian fits are shown as black lines in Fig. $7 \mathrm{~d}$ to $\mathrm{f}$. Due to the lower number of comparisons in these distributions, a resolution of $1^{\circ}$ has been used. The mean $(\mu)$, standard deviation $(\sigma)$, and percentile values for the differences between the DMSP PAPBs and FUV PALBs are summarised in Table 2. In each case, the boundary differences relating to PALBs derived from double Gaussian fits have 

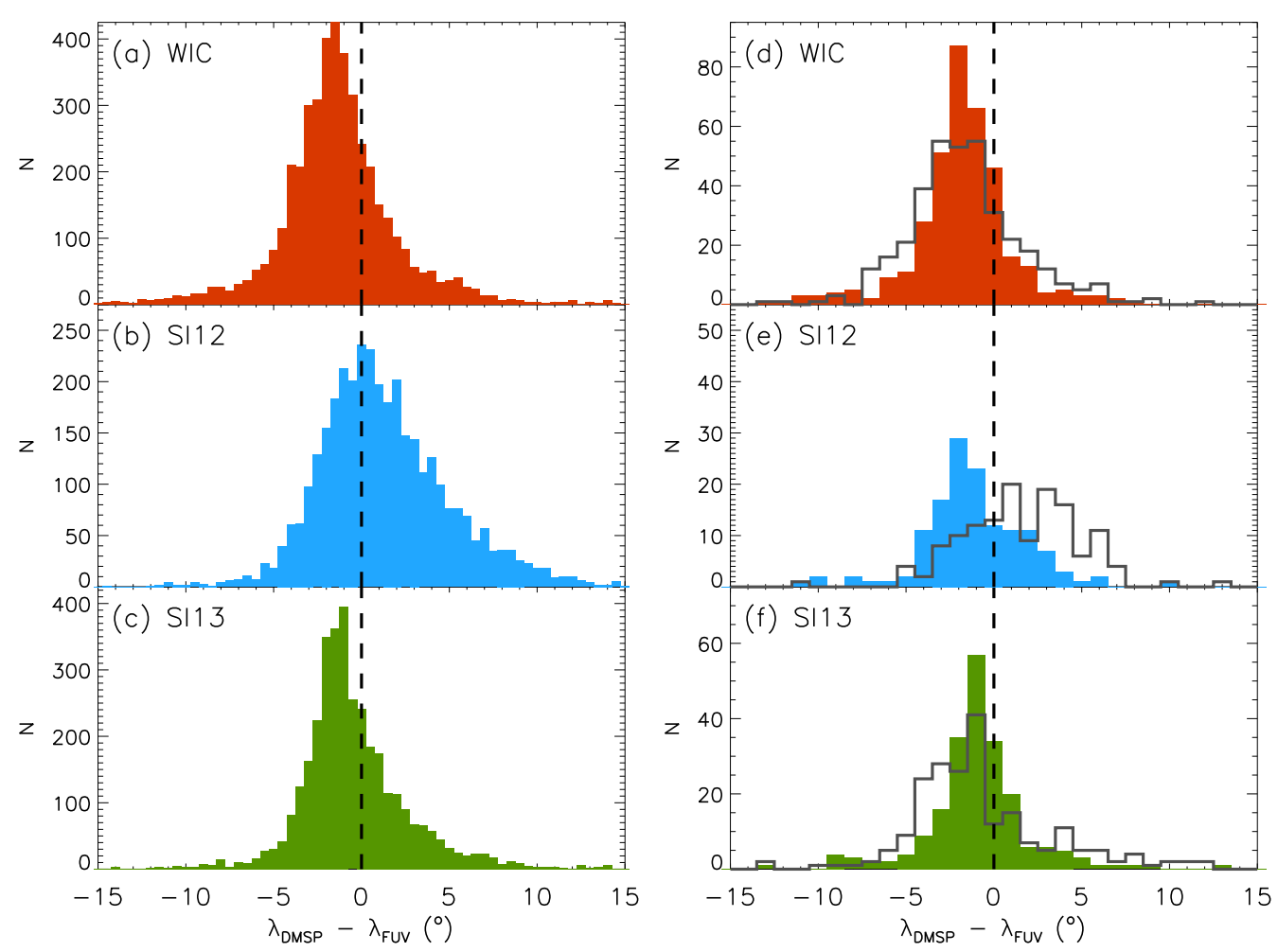

Fig. 7. Distribution of differences between DMSP PAPBs and IMAGE FUV PALBs derived using the method outlined in this paper during 2000 and 2001. From top to bottom, panels show the distribution of differences between PAPBs and PALBs from the WIC, SI12 and SI13 cameras, respectively. Panels (a) to (c) show the distribution of all differences between PAPBs and PALBs at a resolution of $0.5^{\circ}$. Panels $\mathrm{d}$ to $\mathrm{f}$ show the distribution of differences between PAPBs and PALBs derived from double Gaussian $\left(F_{\mathrm{d}}(\lambda)\right.$ ) fits to intensity profiles (solid coloured bars) and PALBs derived from single Gaussian $\left(F_{\mathrm{S}}(\lambda)\right)$ fits to intensity profiles (black line) at a resolution of $1^{\circ}$. For panels (d) to (f), boundaries are only included when PALBs derived from both the single and double Gaussian fits to an intensity profile pass our criteria but the double Gaussian provides the superior fit to the profile.

a smaller standard deviation than those relating to PALBs from single Gaussian fits, showing that the double Gaussian fit yields a boundary more consistent with PAPBs than would have been obtained by a single Gaussian fit. The interquartile range is also lower for PALBs from double Gaussian fits than those from single Gaussian fits for all FUV cameras. It must be noted that the sample here is limited, and is biased by the requirement that both the double and single Gaussians are good fits to the profile, therefore excluding a large majority of cases when the double Gaussian represents a much better fit to the emission profile than a single Gaussian fit that fails the criteria. Hence, the improved correlation with the PAPBs is more subtle here than in reality.

Table 3 lists a summary of the comparisons of DMSP PAPBs and PALBs in each MLT sector taken from each of the IMAGE FUV detectors, including the number of comparisons made $(\mathrm{N})$, and the mode of the distribution of the differences between these boundary locations (M). Due to the low frequency of differences in some MLT sectors, the difference distribution in each MLT sector has been smoothed three times using a boxcar average over five points (e.g., Boakes et al., 2008). The MLT variation of these smoothed modal differences for each FUV detector are shown in Fig. 8a to c (diamond symbols). Error bars show estimates for the standard deviations derived from fitting a Gaussian function to each distribution. To obtain estimates for the expected modal values in the MLT sectors where no DMSP PAPBs are available, we model the MLT variation of the modal differences $(L(\varphi))$ as a second-order harmonic function (e.g., Carbary et al., 2003; Boakes et al., 2008):

$$
L(\varphi)=C_{0}+C_{1} \cos \varphi+D_{1} \sin \varphi+C_{2} \cos 2 \varphi+D_{2} \sin 2 \varphi
$$

where $\varphi$ is the angle associated with each MLT sector in circular coordinates with $0^{\circ}$ at midnight, and increasing with increasing MLT, and $C_{0}, C_{1}, C_{2}, D_{1}$ and $D_{2}$ are coefficients of the fit. We again use the Levenberg-Marquardt least squares fitting routine adapted from Press et al. (1992) to obtain the parameters of the fit. No weighting was applied during fitting. The results of these fits are shown as the continuous curves in Figs. 8a to c. The modal differences estimated from these fits, rounded to the nearest $0.5^{\circ}$, are shown in Table 3 in square brackets for the MLT sectors without DMSP 

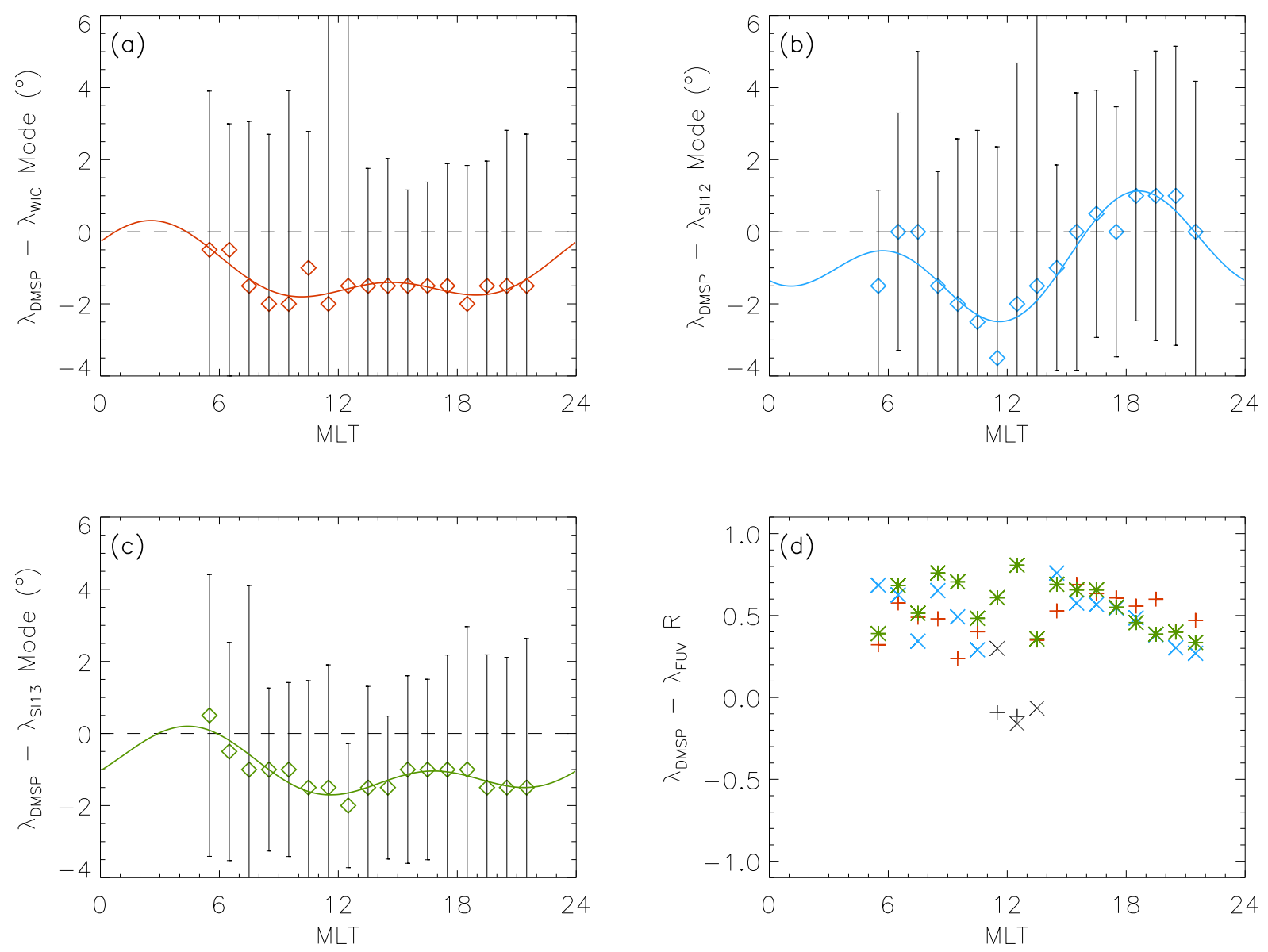

Fig. 8. Comparison of DMSP PAPBs and IMAGE FUV PALBs derived using the method outlined in this paper during 2000 and 2001. Panels (a) to (c) show the modes of the smoothed difference distributions between PAPBs and PALBs from the WIC, SI12 and SI13 cameras, respectively, with a resolution of $0.5^{\circ}$, represented by diamond symbols. The solid lines in each panel show the second-order harmonic fits to the modal distribution values. Error bars show estimates for the standard deviations of the boundary differences. Panel (d) shows the correlation coefficient of PAPBs and PALBs from the WIC (red plus symbols), SI12 (blue cross symbols), and SI13 (green asterisks) FUV cameras in each MLT sector. Grey symbols indicate correlation coefficient values that are not significant to the $95 \%$ confidence level.

data. Figure 8d shows the correlation coefficients for DMSP PAPBs and FUV PALBs in each MLT sector. Coloured symbols show significant correlation coefficients while grey symbols indicate coefficients that are not significant at the $95 \%$ confidence level.

In addition to the differences between DMSP PAPBs and FUV PALBs, Table 3 also lists the (unsmoothed) modal values of the distribution of differences between PALBs obtained from the different FUV cameras and the number of inter-camera comparisons available during May 2000 until August 2002 in each MLT sector. Due to the typically higher numbers of inter-camera comparisons relative to the comparisons with DMSP PAPBs, a resolution of $0.2^{\circ}$ was used for these difference distributions. Inter-camera comparisons are made between successful PALBs within $\pm 15 \mathrm{~s}$ UT and in the same MLT sector.
From the modal differences of the inter-camera comparisons, it can be seen that PALBs obtained from WIC and SI13 images are largely the same, with a slight poleward offset for WIC PALBs of up to $0.6^{\circ}$ across all MLTs. As expected from the offset between electron and proton aurora, the modal difference values indicate that PALBs from SI12 images are typically located poleward of those from WIC and SI13 in the predawn sector and equatorward around dusk.

\subsection{OCB estimate correction}

Carbary et al. (2003) and Boakes et al. (2008) proposed constant offset corrections to minimise the systematic error observed between DMSP PAPBs (thought to provide the most accurate proxy for the OCB) and PALBs derived from auroral images, aiming to improve the accuracy of the PALB as a proxy for the OCB. We use the modal differences between the FUV PALBs and DMSP PAPBs as well 


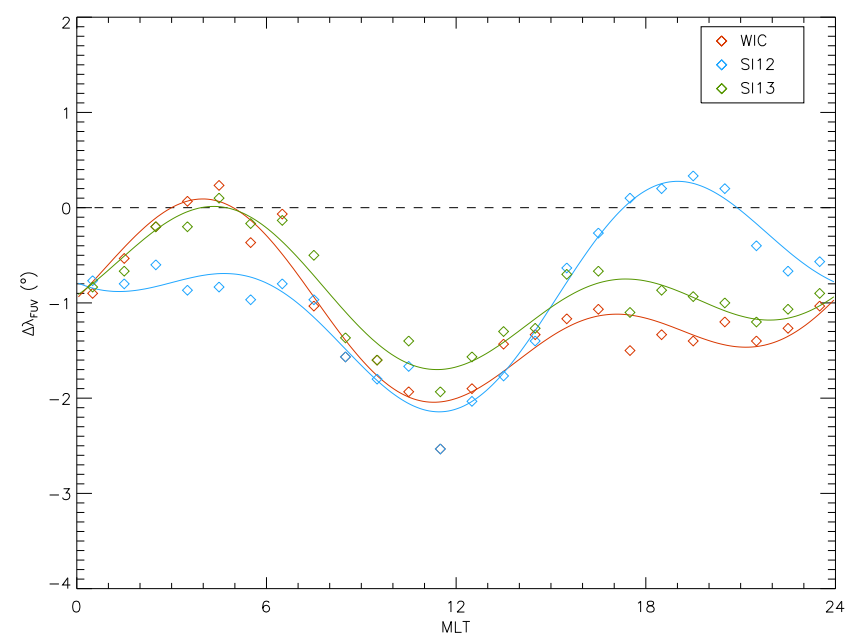

Fig. 9. Correction offset values for PALBs derived from images from the IMAGE FUV WIC, SI12, and SI13 detectors using the method outlined in this paper between May 2000 and August 2002, represented by diamond symbols. The solid lines in each panel show the second-order harmonic fits to these offsets.

as the modes of the inter-camera comparisons (as listed in Table 3 ) to calculate the necessary offsets $\left(\Delta \lambda_{\mathrm{FUV}}\right)$ to correct PALBs from each FUV camera using Eqs. (11) to (13) of Boakes et al. (2008) (Eq. 13 of Boakes et al. (2008) has a typographical error and should read $E-D=\left(-x_{2}-\right.$ $\left.x_{3}-x_{4}-x_{5}-x_{6}\right) / 3$ ). We invert these offset values such that $\lambda_{\mathrm{OCB}}=\lambda_{\mathrm{FUV}}+\Delta \lambda_{\mathrm{FUV}}$. We model the resulting offset values for each FUV camera as a second-order harmonic fit in the form of Eq. (6) (with $\Delta \lambda_{\text {FUV }}$ replacing $L(\varphi)$ ), again using the Levenberg-Marquardt least squares fitting routine adapted from Press et al. (1992). These offset values and fits are shown in Fig. 9, and the coefficients of these fits are also listed in Table 4. An MLT dependence in the systematic offsets is clear from Fig. 9, with PALBs from all cameras being corrected equatorward around local noon and midnight. Around local dawn, PALBs from SI13 and WIC need relatively little correction while PALBs from SI12 are corrected equatorward. Around local dusk, PALBs from SI12 are corrected slightly poleward whereas PALBs from WIC and SI13 are corrected equatorward. The harmonic coefficients presented in Table 4 can be used in Eq. (6) to provide the correction offset $\left(\Delta \lambda_{\mathrm{FUV}}\right)$ for PALB estimates from any camera at any MLT. The correlation coefficients shown in Fig. $8 \mathrm{~d}$ indicate the applicability and suitability of these offsets (the inter-camera correlation coefficients are significant to the $95 \%$ confidence level in all MLT sectors). Hence, these offset corrections should only be used when the correlation coefficients between DMSP PAPBs and FUV PALBs are significant, i.e., WIC corrections should not be used in the 11:00 to 13:00 MLT sector and SI12 corrections should not be used in the 11:00 to 14:00 MLT sector.
Table 4. Harmonic coefficients for correction of PALBs derived from IMAGE FUV images between May 2000 and August 2002.

\begin{tabular}{rrrrrr}
\hline & $C_{0}$ & $C_{1}$ & $D_{1}$ & $C_{2}$ & $D_{2}$ \\
\hline WIC & -1.10 & 0.52 & 0.43 & -0.38 & 0.43 \\
SI12 & -0.88 & 0.66 & -0.49 & -0.57 & -0.04 \\
SI13 & -0.89 & 0.37 & 0.28 & -0.40 & 0.31 \\
\hline
\end{tabular}

\section{Polar cap area estimation}

Assuming a spherical geometry, the PCA can be estimated from the OCB (or the measured proxies) using Eq. (7) (e.g. Carbary et al., 2003; Chisham et al., 2008):

$\mathrm{PCA}=\int_{0}^{2 \pi}\left(R_{\mathrm{E}}+h\right)^{2}\left[1-\sin \left(\lambda_{\mathrm{OCB}}(\phi)\right)\right] d \phi$

where $h$ is the effective altitude of the measurements, $\lambda_{\text {OCB }}$ is the magnetic latitude of the OCB, and $\phi$ is the magnetic longitude. Our corrected PALBs define a proxy measure for the latitude of the OCB at 24 evenly-spaced magnetic longitudes and, hence, the PCA can be estimated from a summation of the bounded areas in each MLT sector $(\varphi)$ using Eq. (8) (e.g. Carbary et al., 2003; Chisham et al., 2008):

$\mathrm{PCA}=\frac{2 \pi\left(R_{\mathrm{E}}+h\right)^{2}}{24} \sum_{i=1}^{24}\left[1-\sin \left(\lambda_{\mathrm{p}}\left(\varphi_{i}\right)+\Delta \lambda\left(\varphi_{i}\right)\right)\right]$

As PALB estimates may not be available for all 24 MLT sectors in an image, a method to estimate the boundaries in the missing sectors must be used. Where PALB estimates are not available for all 24 MLT sectors in an image, a simple linear piece-wise interpolation across the successfully defined boundaries is assumed.

As an example, Fig. 10 shows the intensity counts recorded by each channel of the IMAGE FUV instrument on 28 October 2001 at $\sim 09: 18$ UT in AACGM coordinates. Panels (a) to (c) show the intensity counts recorded by WIC (red), SI12 (blue), and SI13 (green) respectively. Panel d shows the composite intensity counts when the most appropriate camera for each MLT sector is selected, with the colourscale corresponding to that of the selected camera. In the MLT sectors where statistical comparisons with the DMSP PAPBs were available, we determine the most appropriate FUV camera to be the one where PALBs show the highest correlation with PAPBs. For the MLT sectors where PAPBs are not available, we select the camera that typically produces the most poleward boundary of the three based on the modal differences of the inter-camera comparison (i.e., WIC from 22:00 to 01:00 MLT and SI12 from 01:00 to 05:00 MLT). Here, we are making the conservative assumption that all particle precipitation that results in significant auroral luminosity occurs on closed field lines, and hence the OCB must be poleward of all regions of auroral luminosity. In the 22:00 to 00:00 MLT sectors, where PALBs 

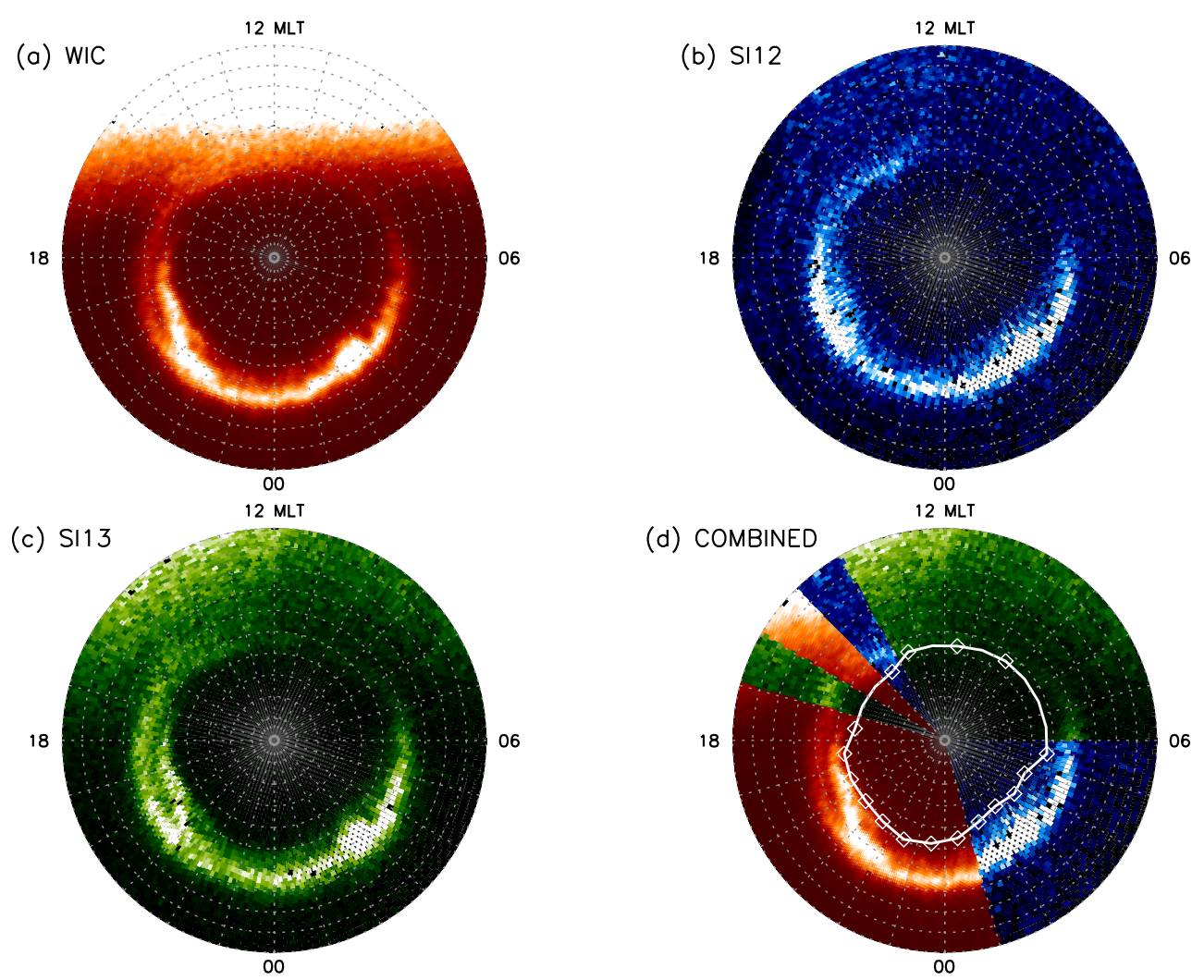

Fig. 10. FUV intensity observed by the IMAGE FUV instrument at 09:18 UT 28th October 2001 in AACGM coordinates. Panels (a) to (c) show the intensity values from the WIC, SI12, and SI13 cameras respectively. Panel (d) shows the composite intensities obtained when selecting the most appropriate camera for each MLT sector. The white diamonds in panel (d) show the OCB location estimates made using the method outlined in this paper from the selected camera. The correction offsets have been added to these OCB locations.

could be taken from either the WIC or SI13 camera, we use WIC data due to its higher image resolution. This instrument selection is in agreement with those used in the 22:00 to 05:00 MLT sectors by Boakes et al. (2008). Overlaid on the composite image are white diamonds indicating the estimates for the OCB locations from the PALBs with systematic offsets corrected for. The white curve illustrates the linear interpolation across the MLT sectors for which no PALB is available. The area enclosed by this curve is considered to be the PCA.

Figure 11 shows PCA estimates made during the period of moderate geomagnetic activity on the morning of 1 February 2001 (as covered in Fig. 2). Panel (a) shows the PCA estimates made during this time period for three different OCB estimation methods: (1) OCBs estimated from PALBs from single Gaussian models of WIC intensity profiles (blue curve), (2) OCBs estimated from PALBs from single Gaussian models of intensity profiles from the best combination of three IMAGE FUV cameras (yellow dashed curve), and (3) OCBs estimated from PALBs from the combination of single and double Gaussian models of intensity profiles from the best combination of the FUV cameras (red curve). OCB correction offsets as outlined in Sect. 4.4 have been applied to the boundaries used in method 3. We have calculated additional OCB correction offsets using PALBs derived from single Gaussian fits only and applied these to the boundaries used in PCA estimation methods 1 and 2. Panel (b) in Fig. 11 shows the percentage differences between the PCAs from method 3 and the other two PCA methods from panel (a). The vertical black dashed lines indicate the times of substorm onset identified by Frey et al. (2004). PCA estimates are made when PALBs are available for at least 10 of the 24 MLT sectors of the FUV image.

From Fig. 11, expansion of the polar cap prior to the substorm activity can be seen, with contraction of the polar cap following the onset of the second substorm for all three PCA estimates. During the initial period of expansion, prior to $\sim 01: 30$ UT, when bifurcation of the auroral oval was evident in Fig. 2, PCAs calculated using methods 1 and 2 are generally overestimated relative to those from method 3 . At times, this overestimation exceeds $20 \%$ of the total PCA. In the mid-phase of the period shown, from $\sim 02: 00 \mathrm{UT}$ until the onset of the second substorm at $\sim 04: 26$ UT, the PCA estimates from methods 2 and 3 are highly similar as during this time bifurcation in the auroral oval was limited. Following the onset of the second substorm, while the auroral 


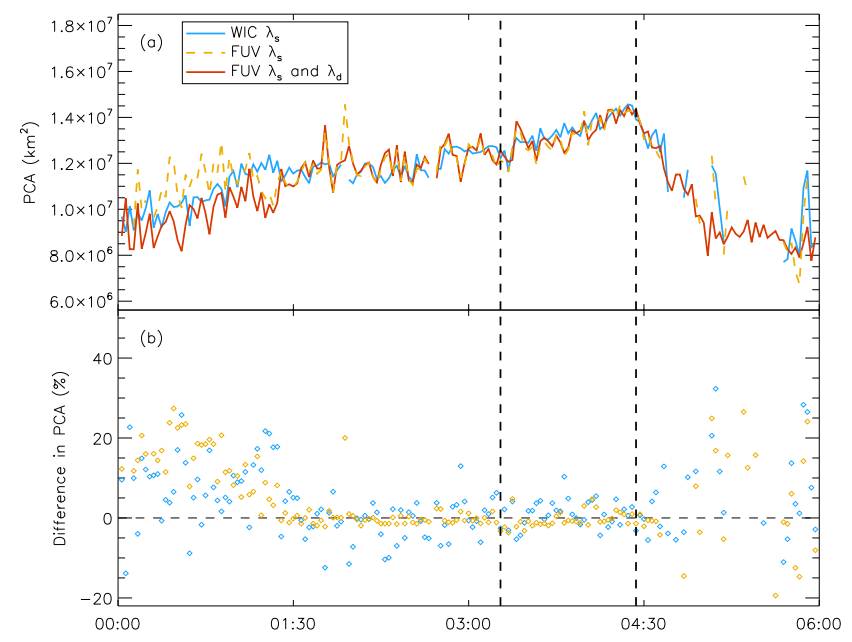

Fig. 11. Polar cap areas estimated from IMAGE FUV auroral boundaries on the 1 February 2001 between 00:00 and 06:00 UT. Panel (a) shows the PCA estimates derived from auroral boundaries obtained using the method outlined in this paper. The areas are calculated from boundaries estimated from single and double Gaussian fits to intensity profiles from the best combination of all three IMAGE FUV cameras (solid red line), from boundaries obtained from single Gaussian fits to intensity profiles from the best combination of all three cameras (dashed yellow line), and from boundaries obtained from single Gaussian fits to intensity profiles from the WIC camera only (solid blue line). Correction offset values have been applied to these auroral boundaries. Panel (b) shows the relative difference in PCA estimated from boundaries from single Gaussian fits from all three FUV cameras (yellow diamonds), and boundaries from single Gaussian fits from the WIC camera (blue diamonds) with respect to area estimates from boundaries derived from both single and double Gaussian fits to intensity profiles from all three FUV cameras. Vertical dashed black lines indicate times of substorm onset from the list outlined in Frey et al. (2004).

oval undergoes contraction, fewer PCA estimates exist when OCBs are derived from fits of a single Gaussian function only. Again, the PCA estimates made from single Gaussian fits only (methods 1 and 2) are frequently substantially different to those from the combined single and double Gaussian fits (method 3).

\section{Conclusions}

Building on the strengths of previous techniques (e.g. Carbary et al., 2003; Mende et al., 2003; Boakes et al., 2008; Gjerloev et al., 2008), we have developed a new, more general, method for estimating the location of auroral oval luminosity boundaries. This technique is fully automated, requiring no prior knowledge of geomagnetic conditions, and can be used to estimate auroral luminosity boundaries during all levels of auroral activity. A case study and statistical analysis demonstrate that the accuracy of auroral luminosity boundary locations can be improved by modelling latitudinal profiles of auroral luminosity intensity as either a single or double Gaussian function compared to modelling these profiles as a single Gaussian function alone. We have identified that a significant percentage of intensity profiles exhibit a degree of bifurcation, with up to $35 \%$ of WIC profiles in nightside MLTs being better modelled by a double Gaussian form, and over 50\% during high geomagnetic activity. Following the techniques of Carbary et al. (2003) and Boakes et al. (2008), we have derived systematic correction values to improve our PALBs as a proxy for the OCB. By applying our technique to a large number of IMAGE FUV images, we have produced a substantial database of OCB location estimates. From the case study of a single storm event where bifurcation is present in the auroral oval, we have demonstrated how these OCB proxies can be used to calculate the PCA and shown that the technique used to derive PALBs from models of a single Gaussian function can produce significantly different PCA estimates than those implementing combined single and double Gaussian modelling. These differences, coupled with the number of successfully estimated PALBs from fits in the form of $F_{\mathrm{d}}(\lambda)$, could have implications for studies where changes in the PCA are used as a proxy for the rate of magnetic reconnection during storm or substorm activity. The dataset that we have compiled opens up the opportunity to measure the rate of reconnection across an unprecedented range of temporal and spatial scales. The auroral boundaries that we have derived from IMAGE data using the technique outlined in this paper are available to download at www.antarctica.ac.uk/bas_research/our_research/az/ magnetic_reconnection/auroral_boundary_data.html

Acknowledgements. NL is supported by the UK Science and Technology Facilities Council grant PP/E002110/1. GC and MPF are supported by the UK Natural Environment Research Council through the Complexity workpackage of the BAS Environmental Change and Evolution Programme. The authors would like to thank the NASA Space Physics Data Facility and National Space Science Data Centre, and the IMAGE FUV team for data usage, processing tools, and advice. The authors would also like to thank Peter Boakes for useful conversations and the Radio Space Plasma Physics group at Leicester University for assistance with data acquisition. NORSTAR $630 \mathrm{~nm}$ MSP data were obtained from the Canadian Space Science Data Portal.

Topical Editor I. A. Daglis thanks J. Wanliss and J. Baker for their help in evaluating this paper.

\section{References}

Akasofu, S.-I. and Kimball, D.: Auroral morphology as shown by all-sky photographs: Arctic and Antarctic, Annals of the International Geophysical Year, 38, 1965.

Baker, J., Clauer, C., Ridley, A., Papitashvili, V., Brittnacher, M., and Newell, P.: The nightside poleward boundary of the auroral oval as seen by DMSP and the Ultraviolet Imager, J. Geophys. Res., 105, 21267-21280, 2000. 
Baker, K. and Wing, S.: A new magnetic coordinate system for conjugate studies at high latitudes, J. Geophys. Res., 94, 91399143, 1989.

Baker, K., Dudeney, J., Greenwald, R., Pinnock, M., Newell, P., Roger, A., Mattin, N., and Meng, C.-I.: HF radar signatures of the cusp and low-latitude boundary layer, J. Geophys. Res., 100, 7671-7695, 1995.

Baker, K., Rodger, A., and Lu, G.: HF-radar observations of the dayside magnetic merging rate: A Geospace Environment Modeling boundary layer campaign study, J. Geophys. Res., 102, 9603-9617, 1997.

Blanchard, G., Lyons, L., Samson, J., and Rich, F.: Locating the polar cap boundary from observations of $6300 \AA$ auroral emission, J. Geophys. Res., 100, 7855-7862, 1995.

Boakes, P. D., Milan, S. E., Abel, G. A., Freeman, M. P., Chisham, G., Hubert, B., and Sotirelis, T.: On the use of IMAGE FUV for estimating the latitude of the open/closed magnetic field line boundary in the ionosphere, Ann. Geophys., 26, 2759-2769, doi:10.5194/angeo-26-2759-2008, 2008.

Brittnacher, M., Fillingim, M., and Parks, G.: Polar cap area and boundary motion during substorms, J. Geophys. Res., 104, 12251-12262, 1999.

Burch, J.: IMAGE mission overview, Space Sci. Rev., 91, 1-14, 2000.

Carbary, J., Sotirelis, T., Newell, P., and Meng, C.-I.: Auroral boundary correlations between UVI and DMSP, J. Geophys. Res., 108, 1018, doi:10.1029/2002JA009378, 2003.

Chisham, G. and Freeman, M. P.: A technique for accurately determining the cusp-region polar cap boundary using SuperDARN HF radar measurements, Ann. Geophys., 21, 983-996, doi:10.5194/angeo-21-983-2003, 2003.

Chisham, G., Pinnock, M., and Rodger, A.: The response of the HF radar spectral width boundary to a switch in the IMF $B_{y}$ direction: Ionospheric consequences of transient dayside reconnection?, J. Geophys. Res., 106, 191-202, 2001.

Chisham, G., Pinnock, M., Coleman, I. J., Hairston, M. R., and Walker, A. D. M.: An unusual geometry of the ionospheric signature of the cusp: implications for magnetopause merging sites, Ann. Geophys., 20, 29-40, doi:10.5194/angeo-20-292002, 2002.

Chisham, G., Freeman, M. P., Coleman, I. J., Pinnock, M., Hairston, M. R., Lester, M., and Sofko, G.: Measuring the dayside reconnection rate during an interval of due northward interplanetary magnetic field, Ann. Geophys., 22, 4243-4258, doi:10.5194/angeo-22-4243-2004, 2004.

Chisham, G., Freeman, M., Abel, G., Lam, M., Pinnock, M., Coleman, I., Milan, S., Lester, M., Bristow, W., Greenwald, R., Sofko, G., and Villain, J.-P.: Remote sensing of the spatial and temporal structure of the magnetopause and magnetotail reconnection from the ionosphere, Rev. Geophys., 46, RG1004, doi: 10.1029/2007RG000223, 2008.

DeJong, A. D., Cai, X., Clauer, R. C., and Spann, J. F.: Aurora and open magnetic flux during isolated substorms, sawteeth, and SMC events, Ann. Geophys., 25, 1865-1876, doi:10.5194/angeo-25-1865-2007, 2007.

Elphinstone, R., Jankowska, K., Murphree, J., and Cogger, L.: The configuration of the auroral distribution for interplanetary magnetic field $B_{Z}$ northward. 1. IMF $B_{x}$ and $B_{y}$ dependencies as observed by the Viking satellite, J. Geophys. Res., 95, 5791-5804,
1990.

Elphinstone, R., Hearn, D., Cogger, L., Murphree, J., Wright, A., Sandahl, I., Ohtani, S., Neweel, P., Klumpar, D., Shapsak, M., Potemra, T., Mursula, K., and Sauvaud, J.: The double oval UV auroral distribution. 2. The most poleward arc system and the dynamics of the magnetotail, J. Geophys. Res., 100, 12093-12102, 1995a.

Elphinstone, R., Murphree, J., Hearn, D., Cogger, L., Sandahl, I., Newell, P., Klumpar, D., Ohtani, S., Sauvaud, J., Potemra, T., Mursula, K., Wright, A., and Shapshak, M.: The double oval UV auroral distribution. 1. Implications for the mapping of auroral arcs, J. Geophys. Res., 100, 12075-12092, 1995 b.

Feldstein, Y. and Galperin, Y. I.: The auroral luminosity structure in the high-latitude upper atmosphere: its dynamics and relationship to the large-scale structure of the Earth's magnetosphere, Rev. Geophys., 23, 217-275, 1985.

Frey, H.: Localized aurora beyond the auroral oval, Rev. Geophys., 45, RG1003, doi:10.1029/2005RG000174, 2007.

Frey, H. and Mende, S.: Substorm onsets as observed by IMAGEFUV, in: Proceedings of the Eigth International Conference on Substorms (ICS-8), edited by: Syrjäsuo, M. and Donovan, E. F., University of Calgaray, Alberta, Canada, 71-75, 2006.

Frey, H., Mende, S., and Angelopoulos, V.: Substorm onset observations by IMAGE-FUV, J. Geophys. Res., 109, A10304, doi: 10.1029/2004JA010607, 2004.

Gibson, W., Burch, J., Scherrer, J., Tapley, M., Killough, R., Volpe, F., Davis, W., Vaccarello, D., Grismore, G., Sakkas, D., and Housten, S.: The IMAGE observatory, Space Sci. Rev., 91, 15$50,2000$.

Gjerloev, J., Hoffman, R., Sigwarth, J., and Frank, L.: Statistical description of the bulge-type auroal substorm in the far ultraviolet, J. Geophys. Res., 112, A07213, doi:10.1029/2006JA012189, 2007.

Gjerloev, J., Hoffman, R., Sigwarth, J., Frank, L., and Baker, J.: Typical auroral substorm: A bifurcated oval, J. Geophys. Res., 113, A03211, doi:10.1029/2007JA012431, 2008.

Hardy, D., Schmitt, L., Gussenhoveann, M., Marshall, F., Yeh, H., Schumaker, T., Huber, A., and Pantazis, J.: Precipitating electron and ion detectors (SSJ/4) for the block 5D/flights 6-10 DMSP satellites: Calibration and data presentation, in: Environmental Research Papers, 902, Air Force Geophysics Laboratory, Hanscom Air Force Base, MA, 1984.

Hoffman, R. A., Gjerloev, J. W., Frank, L. A., and Sigwarth, J. W.: Are there optical differences between storm-time substorms and isolated substorms?, Ann. Geophys., 28, 1183-1198, doi:10.5194/angeo-28-1183-2010, 2010.

Hubert, B., Miolan, S., Grocott, A., Blockx, C., Cowley, S., and Gérard, J.-C.: Dayside and nightside reconnection rates inferred from IMAGE FUV and Super Dual Auroral Radar Network data, J. Geophys. Res., 111, A03217, doi:10.1029/2005JA011140, 2006.

Iijima, T. and Potemra, T.: Large-scale characteristics of fieldaligned currents associated with substorms, J. Geophys. Res., 83, 599-615, 1978.

Immel, T., Craven, J., and Nicholas, A.: An empiracal model of the OI FUV dayglow from DE-1 images, J. Atmos. Sol.-Terr. Phy., 62, 47-64, 2000.

Kauristie, K., Weygand, J., Pulkkinen, T., Murphree, J., and Newell, P.: Size of the auroral oval: UV ovals and precipitation bound- 
aries compared, J. Geophys. Res., 104, 2321-2331, 1999.

Makita, K. and Meng, C.-I.: Average electron precipitation patterns and visual aurora characteristics during geomagnetic quiescence, J. Geophys. Res., 89, 2861-2872, 1984.

Makita, K., Meng, C.-I., and Akasofu, S.-I.: The shift of the auroral electron precipitation boundaries in the dusk-dawn sector in association with geomagnetic activity and interplanetary magnetic field, J. Geophys. Res., 88, 7967-7981, 1983.

Meier, R.: Ultraviolet spectroscopy and remote sensing of the upper atmosphere, Space Sci. Rev., 58, 1-185, 1991.

Mende, S., Heetderks, H., Frey, H., Lampton, M., Geller, S., Abiad, R., Siegmund, O., Tremsin, A., Spann, J., Dougani, H., Fuselier, S., Magoncelli, A., Bumala, M., Murphree, S., and Trondsen, T.: Far ultraviolet imaging from the IMAGE spacecraft. 2. Wideband FUV imaging, Space Sci. Rev., 91, 271-285, 2000a.

Mende, S., Heetderks, H., Frey, H., Lampton, M., Geller, S., Habraken, S., Renotte, E., Jamar, C., Rochus, P., Spann, J., Fuselier, S., Gerard, J.-C., Gladstone, R., Murphree, S., and Cogger, L.: Far ultraviolet imaging from the IMAGE spacecraft. 1. System design, Space Sci. Rev., 91, 243-270, 2000 b.

Mende, S., Heetderks, H., Frey, H., Stock, J., Lampton, M., Geller, S., Abiad, R., Siegmund, O., Habraken, S., Renotte, E., Jamar, C., Rochus, P., Gerard, J.-C., Sigler, R., and Lauche, H.: Far ultraviolet imaging from the IMAGE spacecraft. 3. Spectral imaging of the Lyman- $\alpha$ and OI $135.6 \mathrm{~nm}$, Space Sci. Rev., 91, 287318, 2000c.

Mende, S., Frey, H., Morsony, B., and Immel, T.: Statistical behaviour of proton and electron auroras during substorms, J. Geophys. Res., 108, 1339, doi:10.1029/2002JA009751, 2003.

Meurant, M., Gérard, J.-C., Hubert, B., Coumans, V., Blockx, C., Østgaard, N., and Mende, S.: Dynamics of global scale electron and proton precipitation induced by a solar wind pressure pulse, Geophys. Res. Lett., 30, 2032, doi:10.1029/2003GL018017, 2003.

Milan, S. E. and Lester, M.: A classification of spectral populations observed in HF radar backscatter from the E region auroral electrojets, Ann. Geophys., 19, 189-204, doi:10.5194/angeo-19189-2001, 2001.

Milan, S. E., Lester, M., Cowley, S. W. H., Moen, J., Sandholt, P. E., and Owen, C. J.: Meridian-scanning photometer, coherent HF radar, and magnetometer observations of the cusp: a case study, Ann. Geophys., 17, 159-172, doi:10.1007/s00585-999-0159-5, 1999.
Milan, S. E., Lester, M., Cowley, S. W. H., Oksavik, K., Brittnacher, M., Greenwald, R. A., Sofko, G., and Villain, J.-P.: Variations in the polar cap area during two substorm cycles, Ann. Geophys., 21, 1121-1140, doi:10.5194/angeo-21-1121-2003, 2003.

Mishin, V.: The magnetogram inversion technique and some applications, Space Sci. Rev., 53, 83-163, 1990.

Mishin, V., Bazarzhapov, A., Saifudinova, T., Lunyushkin, S., Shirapov, S. S., Woch, J., Eliasson, L., Opgenoorth, H., and Murphree, J.: Different methods to determine the polar cap area, J. Geomagn. Geoelectr., 44, 1207-1214, 1992.

Newell, P., Burke, W., Sánchez, E., Meng, C.-I., Greenspan, M., and Clauer, C.: The low-latitude boundary layer and the boundary plasma sheet at low altitude: Prenoon precipitation regions and convection reversal boundaries, J. Geophys. Res., 96, 2101321023, 1991.

Newell, P., Feldstein, Y., Galperin, Y., and Meng, C.-I.: Morphology of nightside precipitation, J. Geophys. Res., 101, 10737 10748, 1996.

Newell, P., Xu, D., Meng, C.-I., and Kivelson, M.: Dynamical polar cap: A unifying approach, J. Geophys. Res., 102, 127-139, 1997.

Newell, P., Liou, K., and Wilson, G.: Polar cap particle precipitation and aurora: Review and commentary, J. Atmos. Solar-Terr. Phys., 71, 199-215, doi:10.1016/j.jastp.2009.11.004, 2009.

Pinnock, M., Chisham, G., Coleman, I. J., Freeman, M. P., Hairston, M., and Villain, J.-P.: The location and rate of dayside reconnection during an interval of southward interplanetary magnetic field, Ann. Geophys., 21, 1467-1482, doi:10.5194/angeo-211467-2003, 2003.

Press, W., Teukolsky, S., Vetterling, W., and Flannery, B.: Numerical Recipes in C. The Art of Scientific Computing, Cambridge University Press, 2nd edn., 1992.

Sandholt, P., Farrugia, C., Øieroset, M., Stauning, P., and Denig, W.: Auroral activity associated with unsteady magnetospheric erosion: Observations on December 18, 1990, J. Geophys. Res., 103, 2309-2317, 1998.

Siscoe, G. and Huang, T.: Polar cap inflation and deflation, J. Geophys. Res., 90, 543-547, 1985.

Sotirelis, T. and Newell, P.: Boundary-oriented electron precipitation model, J. Geophys. Res., 105, 18655-18673, 2000.

Vampola, A.: Access of solar electrons to closed field lines, J. Geophys. Res., 76, 36-43, 1971. 